\title{
Taylor-Couette flow with axial oscillations of the inner cylinder: Floquet analysis of the basic flow
}

\author{
By F. MARQUES ${ }^{1}$ AND J. M. LOPEZ $Z^{2}$ \\ ${ }^{1}$ Departament de Física Aplicada, Universitat Politècnica de Catalunya, Jordi Girona Salgado s/n, \\ Mòdul B4 Campus Nord, 08034 Barcelona, Spain \\ ${ }^{2}$ Department of Mathematics and Earth System Science Center, The Pennsylvania State \\ University, University Park, PA 16802, USA
}

(Received 2 December 1996 and in revised form 11 March 1997)

The linear stability of the flow in the annular gap between two infinitely long cylinders, driven by the constant rotation and harmonic oscillation in the axial direction of the inner cylinder, is analysed using Floquet theory. Closed form solutions for the basic flow are derived, both with and without the presence of endwalls. Recent experiments and theory using the narrow gap approximation have shown that the axial oscillations significantly stabilize the flow with respect to centrifugal instabilities. However, the agreement has only been qualitative. The present analysis reproduces the experimental results to well within experimental uncertainty. We have identified the major source of discrepency with the previous theory to be due to the lack of global endwall effects enforcing a net zero axial mass flux. Analysis of the basic flow indicates that the stabilization is primarily due to waves of azimuthal vorticity propagating inwards from the cylinder boundary layers due to the axial oscillations.

\section{Introduction}

The response to external time-periodic forcing of flows which are susceptible to instabilities has received much attention (e.g. see Davis 1976) because of their technological importance as well as from a fundamental point of view their rich dynamics. In general, these systems are very complicated and it is worthwhile studying relatively simple generic problems in order to isolate particular mechanisms and to develop and test hypotheses governing their behaviour. The Taylor-Couette flow, consisting of an incompressible fluid held between two concentric cylinders and driven by the rotation of one or both of the cylinders, is a good model problem for such studies. The basic flow solution is known in closed form and its bifurcation structure has been studied extensively (e.g. see Swinney \& Gollub 1985; Chossat \& Iooss 1994). Temporal modulations of the Taylor-Couette flow have also been studied extensively (e.g. Donnelly, Reif \& Suhl 1962; Donnelly 1964; Carmi \& Tustaniwskyj 1981; Barenghi \& Jones 1989; Murray, McFadden \& Coriell 1990). These have only studied modulations of the angular velocity of the cylinders, where the modulations either stabilize or destabilize the onset of Taylor vortex flow, depending on the precise nature of the modulation. However, the degree of stabilization or destabilization has in all cases been found to be small.

Recently, temporal modulations due to axial oscillations of the inner cylinder have attracted attention due to the large degree of stabilization achievable. Hu \& Kelly (1995) considered various time-modulations of the Taylor-Couette flow, including 
cases where the outer cylinder remains stationary and the inner cylinder rotates steadily and translates sinusoidally in the axial direction. Their theoretical study was performed in the narrow gap limit, where curvature effects are neglected. In the narrow gap limit, they derive a closed form solution for the basic flow (independent of the axial and azimuthal directions), and study its stability using Floquet theory. Their results showed a significant stabilizing effect in the presence of oscillating shear.

Weisberg, Smits \& Kevrekidis (1997) studied experimentally for the first time Taylor-Couette flow with axial oscillations of the inner cylinder. The motivation was to investigate transition control strategies based on the stabilization observed in the linear analysis of $\mathrm{Hu} \&$ Kelly (1995). While the experiments and linear theory both showed significant stabilizations due to the axial oscillations, the agreement was only qualitative. The degree of relative stabilization differed between the two studies by a large factor, and the difference was largest for small frequencies and large amplitudes of the axial oscillations. Barenghi \& Jones (1989) and Murray, et al. (1990) give sound physical arguments, based on their considerations of Taylor-Couette flow with a modulation of the cylinder angular velocities, that in the limit of small frequency and large amplitude, perturbations can grow to a level invalidating linearization of the problem during some parts of the oscillation period.

It is not clear whether the quantitative discrepency between Hu \& Kelly's linear results and Weisberg et al.'s experimental results are attributable to a failure of the linear assumption, or to idealizations in the model (i.e. narrow gap and infinite cylinder lengths), or to endwall effects, or a combination of some of these. Here, we also consider the stability of Taylor-Couette flow with axial oscillations of the inner cylinder using Floquet theory, but include a finite gap (curvature) and model the global effects of endwalls by introducing a constant axial pressure gradient. We retain the idealization of infinitely long cylinders. We achieve agreement to well within experimental uncertainty $(<3 \%)$ with all the data of Weisberg et al. corresponding to the onset of Taylor vortex flow. Our study indicates that at the radius ratio of 0.905 employed in the experiment, curvature effects are not very important. The global effects of the endwalls, introduced through the constraint of zero axial mass flux, account for most of the discrepancy between the experiments and the previous theoretical results. The theory of Hu \& Kelly considered an open system, whereas the experiments of Weisberg et al. had endwalls affixed to the stationary outer cylinder with seals that allowed the axial motion of the inner cylinder without leakage of fluid from the annular region. At low frequency of oscillations, the endwall effects become more important, and with just these effects included in the linear theory we obtain excellent agreement with the experiments. An analysis of our derived closed form solution of the time-periodic basic flow indicates that the stabilization effect is due to waves of azimuthal vorticity propagating into the interior from the inner-cylinder boundary layer, and when endwall effects are included, waves of azimuthal vorticity also propagate in from the outer cylinder.

We also note that in narrow windows of parameter space, with large amplitude and small frequency of the axial oscillations, the bifurcation from the basic state is nonaxisymmetric and competition between different azimuthal and axial modes becomes increasingly important. Also, the nature of the frequency going to the zero limit is highly singular, and this raises interesting issues concerning the mathematical and computational aspects of this problem. 


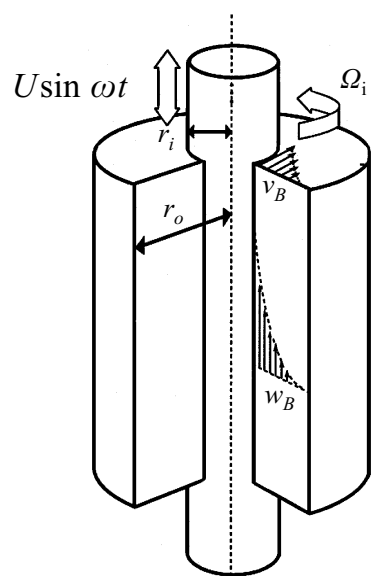

FIGURE 1. Schematic of Taylor-Couette flow with axial oscillations of the inner cylinder.

\section{Basic flow}

Taylor-Couette flow is the term used to describe fluid motion between two concentric rotating cylinders. Here we consider the case where the inner cylinder also moves axially with speed $U \sin \Omega t$. The cylinders, of inner radius $r_{i}$ and outer radius $r_{o}$, can be independently rotated at angular velocities $\Omega_{i}$ and $\Omega_{o}$, respectively. The annular gap between the cylinders is $d=r_{o}-r_{i}$. In the axial direction the cylinders are unbounded, and we assume the flow is periodic in the axial direction. A schematic of the flow is given in figure 1. The independent non-dimensional parameters governing this problem are the radius ratio $e=r_{i} / r_{o}$ which fixes the geometry of the annulus, the Couette-flow Reynolds numbers $R e_{i}=d r_{i} \Omega_{i} / v$ and $R e_{o}=d r_{o} \Omega_{o} / v$, where $v$ is the kinematic viscosity of the fluid, the axial Reynolds number $R e_{a}=d U / v$ measuring the amplitude of the velocity of the inner-cylinder axial oscillations, and the non-dimensional frequency of these oscillations $\omega=d^{2} \Omega / v$.

In order to compare with experiments and also with some previous work, two different situations are considered in this paper. In both, the basic flow velocity field is independent of the axial direction, but in one case the axial pressure gradient is zero (open flow) and in the other non-zero (enclosed flow). The non-zero axial pressure gradient in the enclosed flow case represents the presence of endwalls and allows us to enforce a net zero axial mass flux at any instant in time, not only for the base flow but also for the perturbed flow. The only experiments on Taylor-Couette flow with axial oscillations of the inner cylinder known to us are those of Weisberg et al. (1997) which were carried out in an annulus with fixed endwalls. The large-scale endwall effects are included in the present analysis via the non-zero axial pressure gradient. Such an axial pressure gradient has been implemented by Ali \& Weidman (1993) in the linear analysis of Taylor-Couette flow with the inner cylinder travelling axially with a constant speed and by Edwards et al. (1991) and Sanchez, Crespo \& Marques (1993) for nonlinear computations of the spiral modes in Taylor-Couette flow. Note that we still have the idealization that the cylinders are infinitely long and that the flow has a continuous spectrum of axial wavenumbers in both the open and enclosed flow cases.

All variables are rendered dimensionless using $d, d^{2} / v$, and $v^{2} / d^{2}$ as units for space, time, and the reduced pressure $(p / \rho)$. The non-dimensional Navier-Stokes equations are

$$
\partial_{t} \boldsymbol{v}+\boldsymbol{v} \cdot \nabla \boldsymbol{v}=-\nabla p+\Delta \boldsymbol{v}, \quad \nabla \cdot \boldsymbol{v}=0 .
$$


The boundary conditions for the general flow described above are

$$
\begin{gathered}
u\left(r_{i}\right)=u\left(r_{o}\right)=0, \\
v\left(r_{i}\right)=R e_{i}, \quad v\left(r_{o}\right)=R e_{o}, \\
w\left(r_{i}, t\right)=R e_{a} \sin \omega t, \quad w\left(r_{o}\right)=0,
\end{gathered}
$$

where $(u, v, w)$ are the physical components of the velocity in cylindrical coordinates $(r, \theta, z)$, and $r_{i}=e /(1-e), r_{o}=1 /(1-e)$. In the enclosed case, we have an additional constraint enforcing net zero axial mass flux

$$
\int_{S} w(r, \theta, z, t) r \mathrm{~d} r \mathrm{~d} \theta=0,
$$

where $S$ is any constant- $z$ section. The assumption that the flow is periodic in the axial direction usually means that both the velocity field and the pressure are periodic. But in fact the pressure appears in the Navier-Stokes equations only as a gradient, and so it is possible to have an axially periodic pressure gradient while the pressure itself is not. In particular, the pressure may include a term linear in $z$. This term appears in the Navier-Stokes equations as an overall pressure gradient depending only on time. We express the pressure as $p=p_{1}+p_{2} z$, where $p_{1}(r, \theta, z, t)$ is periodic in the axial direction and $p_{2}(t)$ is the overall axial pressure gradient, depending only on time. This additional degree of freedom is used to enforce the constraint (2.5). The open flow case corresponds to $p_{2}=0$.

We look for a basic flow solution that is axisymmetric and independent of the axial coordinate $z$, but time dependent due to the external forcing. The Navier-Stokes equations and incompressibility condition reduce to

$$
\begin{aligned}
& \partial_{t} u+u \mathrm{D} u-v^{2} / r=-\mathrm{D} p_{1}+\mathrm{DD}_{+} u \\
& \partial_{t} v+u \mathrm{D} v+u v / r=\mathrm{DD}_{+} v \\
& \partial_{t} w+u \mathrm{D} w=-p_{2}+\mathrm{D}_{+} \mathrm{D} w \\
& \mathrm{D}_{+} u=0
\end{aligned}
$$

where $\mathrm{D}=\partial_{r}$ and $\mathrm{D}_{+}=\mathrm{D}+1 / r$. The incompressibility condition (2.9) together with the boundary condition (2.2) give $u=0$, and equations (2.6)-(2.8) reduce to

$$
\begin{aligned}
& \mathrm{D} p_{1}=v^{2} / r, \\
& \partial_{t} v=\mathrm{DD}_{+} v, \\
& \partial_{t} w=\mathrm{D}_{+} \mathrm{D} w-p_{2} .
\end{aligned}
$$

Equation (2.11) is immediately solved with the boundary conditions (2.3) and gives the well-known basic solution for Couette flow

$$
v(r)=\frac{r_{i} r_{o}}{r_{o}^{2}-r_{i}^{2}}\left[\left(\frac{r_{o}}{r}-\frac{r}{r_{o}}\right) R e_{i}+\left(\frac{r}{r_{i}}-\frac{r_{i}}{r}\right) R e_{o}\right],
$$

where we have used the fact that the transients in $v(r, t)$ vanish, leaving only the steady-state solution. Equation (2.10) with (2.13) gives the pressure $p_{1}$, leaving only the $w$-equation to be solved.

In the open flow case, $p_{2}=0$ and the constraint (2.5) does not apply. Equation (2.12) is linear and the boundary condition (2.4) is harmonic in $t$, so we look for a complex solution of the form

$$
w(r, t)=R e_{a} \operatorname{Im}\left(f(r) \mathrm{e}^{\mathrm{i} \omega t}\right)=\operatorname{Re}_{a} W(r) \sin (\omega t+\alpha(r)),
$$


where $W$ and $\alpha$ are the modulus and phase of $f$. The equation for $f$ is

$$
f^{\prime \prime}+\frac{1}{r} f^{\prime}-\mathrm{i} \omega f=0, \quad f\left(r_{i}\right)=1, f\left(r_{o}\right)=0,
$$

whose general solution is a linear combination of the modified Bessel functions of order zero $I_{0}(y), K_{0}(y)$ with complex argument $y=\omega^{1 / 2} r \mathrm{e}^{\mathrm{i} \pi / 4}$. Taking into account the boundary conditions for $f$, the desired solution is

$$
f(r)=\left|\begin{array}{cc}
I_{0}(y) & K_{0}(y) \\
I_{0}\left(y_{o}\right) & K_{0}\left(y_{o}\right)
\end{array}\right| /\left|\begin{array}{cc}
I_{0}\left(y_{i}\right) & K_{0}\left(y_{i}\right) \\
I_{0}\left(y_{o}\right) & K_{0}\left(y_{o}\right)
\end{array}\right|,
$$

where $y_{i}$ and $y_{o}$ are the values of $y$ at $r=r_{i}$ and $r_{o}$ respectively. The real and imaginary parts of $I_{0}(y)$ and $K_{0}(y)$, called Kelvin functions, as well as their modulus and phase, are special functions whose properties can be found in Abramowitz \& Stegun (1972), along with tables and polynomial approximations useful for their numerical computation.

The vorticity vector $\omega=(\xi, \eta, \zeta)$ of the basic flow is

$$
\boldsymbol{\omega}=\left(0,-\mathrm{D} w, \mathrm{D}_{+} v\right)=(0,-\mathrm{D} w, \zeta) .
$$

The axial vorticity is a constant depending on the angular velocities of the cylinders, $\zeta=2\left(r_{o} R e_{o}-r_{i} R e_{i}\right) /\left(r_{i}+r_{o}\right)$. The azimuthal vorticity $\eta$ is a function of $r$ and $t$ given by

$$
\eta(r, t)=R e_{a} \operatorname{Im}\left(g(r) \mathrm{e}^{\mathrm{i} \omega t}\right)=R e_{a} G(r) \sin (\omega t+\beta(r)),
$$

where $g(r)=-f^{\prime}(r)$ is given by

$$
g(r)=-\omega^{1 / 2} \mathrm{e}^{\mathrm{i} \pi / 4}\left|\begin{array}{cc}
I_{1}(y) & -K_{1}(y) \\
I_{0}\left(y_{o}\right) & K_{0}\left(y_{o}\right)
\end{array}\right| /\left|\begin{array}{cc}
I_{0}\left(y_{i}\right) & K_{0}\left(y_{i}\right) \\
I_{0}\left(y_{o}\right) & K_{0}\left(y_{o}\right)
\end{array}\right| .
$$

The azimuthal vorticity $\eta$ is now a combination of the modified Bessel functions of order one $I_{1}(y), K_{1}(y)$, whose real and imaginary parts, as well as their modulus and phase, can also be found in Abramowitz \& Stegun (1972).

For the enclosed flow we must solve (2.12) for $w$ and $p_{2}$. Using (2.14) and the corresponding expression for $p_{2}$

$$
p_{2}(t)=R e_{a} \operatorname{Im}\left(p_{0} \mathrm{e}^{\mathrm{i} \omega t}\right),
$$

with $p_{0}$ a complex constant, the equation for $f$ is now

$$
f^{\prime \prime}+\frac{1}{r} f^{\prime}-\mathrm{i} \omega f=p_{0}, \quad f\left(r_{i}\right)=1, f\left(r_{o}\right)=0, \int_{r_{i}}^{r_{o}} r f(r) \mathrm{d} r=0 .
$$

The last condition comes from the zero axial mass flow (2.5). The solution is a linear combination of the modified Bessel functions of order zero $I_{0}(y), K_{0}(y)$ plus the particular solution $\mathrm{i} p_{0} / \omega$ :

$$
f(r)=A I_{0}(y)+B K_{0}(y)+\mathrm{i} p_{0} / \omega .
$$

$A, B, p_{0}$ are determined by the three conditions in (2.21). Using the fact that

$$
\begin{aligned}
& \int_{r_{i}}^{r_{o}} r I_{0}(y) \mathrm{d} r=\omega^{-1 / 2} \mathrm{e}^{-\mathrm{i} \pi / 4}\left[r_{o} I_{1}\left(y_{o}\right)-r_{i} I_{1}\left(y_{i}\right)\right], \\
& \int_{r_{i}}^{r_{o}} r K_{0}(y) \mathrm{d} r=-\omega^{-1 / 2} \mathrm{e}^{-\mathrm{i} \pi / 4}\left[r_{o} K_{1}\left(y_{o}\right)-r_{i} K_{1}\left(y_{i}\right)\right],
\end{aligned}
$$


(a)
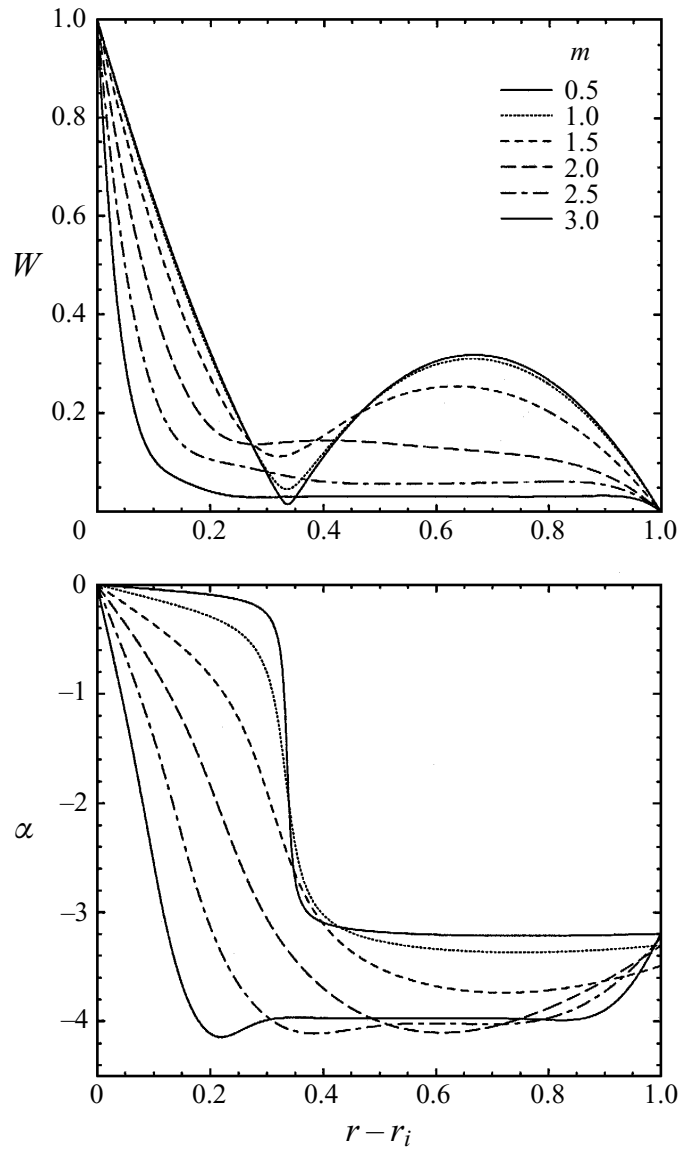

(b)
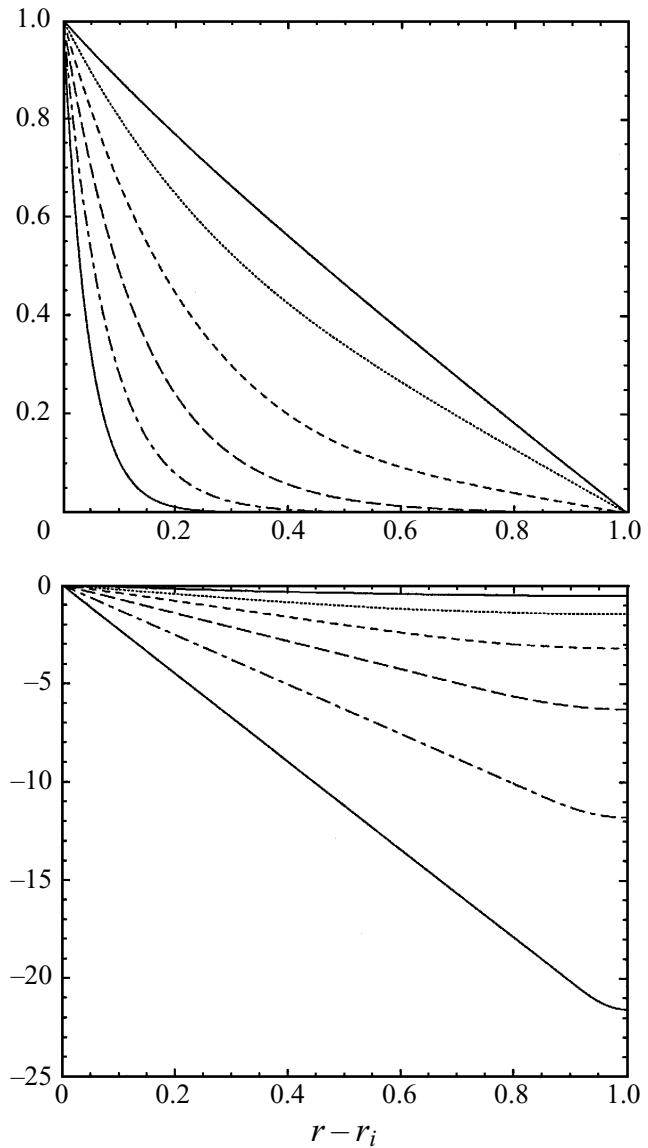

Figure 2. Profiles of the axial velocity amplitude $W(r)$ and phase $\alpha(r)$ of the basic flow solutions for $(a)$ the enclosed flow and $(b)$ the open flow cases, at various oscillation frequencies $\omega=10^{m}$, for $m$ as indicated.

and after some algebra we obtain

$$
\begin{gathered}
f(r)=\frac{1}{D E T}\left|\begin{array}{ccc}
I_{0}(y) & K_{0}(y) & 1 \\
I_{0}\left(y_{o}\right) & K_{0}\left(y_{o}\right) & 1 \\
r_{o} I_{1}\left(y_{o}\right)-r_{i} I_{1}\left(y_{i}\right) & -r_{o} K_{1}\left(y_{o}\right)+r_{i} K_{1}\left(y_{i}\right) & a
\end{array}\right|, \\
p_{0}=\frac{1}{D E T}\left|\begin{array}{cc}
I_{0}\left(y_{o}\right) & K_{0}\left(y_{o}\right) \\
r_{o} I_{1}\left(y_{o}\right)-r_{i} I_{1}\left(y_{i}\right) & -r_{o} K_{1}\left(y_{o}\right)+r_{i} K_{1}\left(y_{i}\right)
\end{array}\right|, \\
D E T=\left|\begin{array}{ccc}
I_{0}\left(y_{i}\right) & K_{0}\left(y_{i}\right) & 1 \\
I_{0}\left(y_{o}\right) & K_{0}\left(y_{o}\right) & 1 \\
r_{o} I_{1}\left(y_{o}\right)-r_{i} I_{1}\left(y_{i}\right) & -r_{o} K_{1}\left(y_{o}\right)+r_{i} K_{1}\left(y_{i}\right) & a
\end{array}\right|,
\end{gathered}
$$

where $a=\omega^{1 / 2} \mathrm{e}^{\mathrm{i} \pi / 4}\left(r_{i}+r_{o}\right) / 2$. The azimuthal vorticity $\eta$ is easily obtained from (2.18), where $g(r)=-f^{\prime}(r)$. Equations (2.16) and (2.25) are two families of time-periodic exact solutions of the Navier-Stokes equations.

The amplitude $W(r)$ and phase $\alpha(r)$ of the axial velocity in (2.14) are displayed in figure 2 for various $\omega$. The differences between the open and enclosed flows are 
(a)
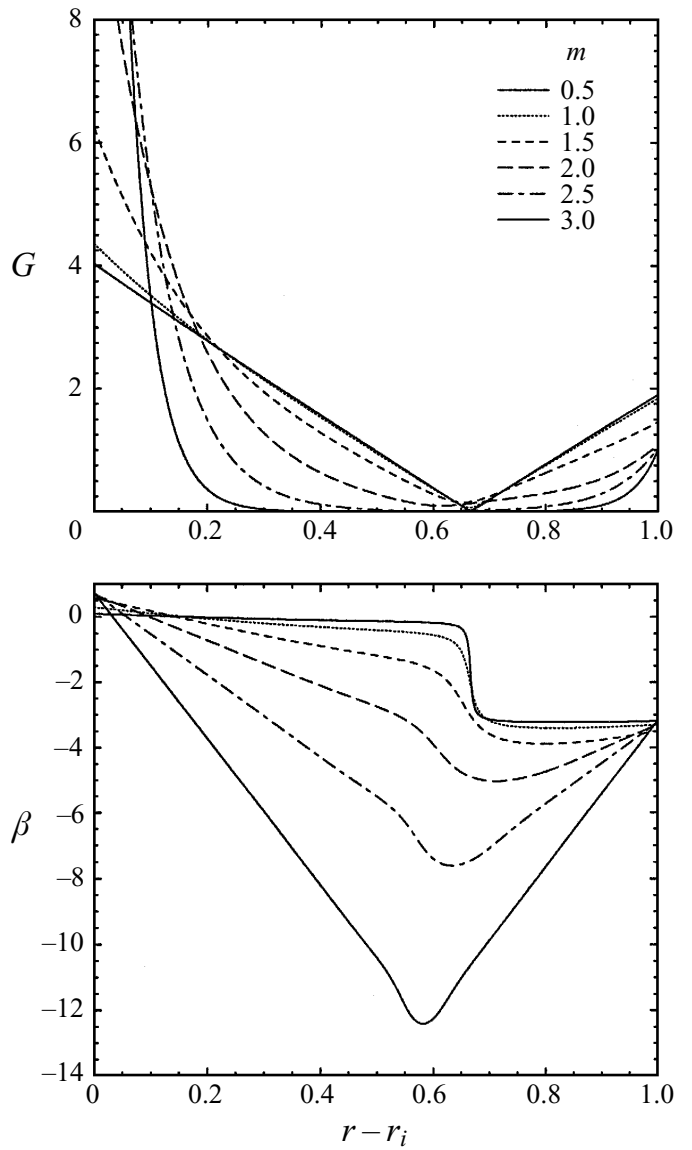

(b)

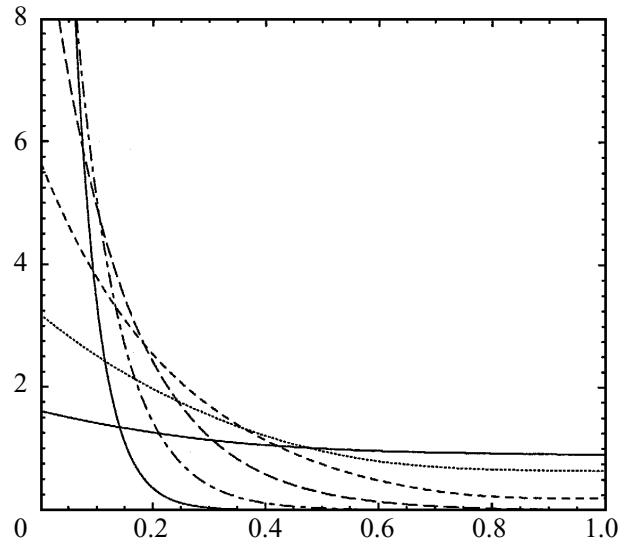

FIGURE 3. Profiles of the azimuthal vorticity amplitude $G(r)$ and phase $\beta(r)$ of the basic flow solutions for $(a)$ the enclosed flow and $(b)$ the open flow cases, at various oscillation frequencies $\omega=10^{m}$, for $m$ as indicated.

striking. In the open flow the velocity amplitude shows a monotone decrease through the gap, almost linear for $\omega \sim 1$, and exponential for $\omega \gg 1$. In the large- $\omega$ case, the axial movement of the fluid is confined to a boundary layer near the inner cylinder. The velocity phase is linear in $r, \alpha(r)=-\tilde{k} r$, showing that the axial velocity induced by the oscillation of the inner cylinder is a radial wave that propagates from the inner to the outer cylinder. The time-dependent part of (2.14) is a function of $\omega t-\tilde{k} r$, and the corresponding wave velocity is $\omega / \tilde{k}$. In the enclosed flow, a return flow appears in the outer region of the annular gap. The presence of this return flow is indicated by a change of $\pi$ in the phase $\alpha(r)$ of the axial velocity. The axial velocity for $\omega \gg 1$ does not go to zero but approaches a value independent of $r$ in the region between the inner-cylinder and the outer-cylinder boundary layers. The return flow has a constant phase corresponding to a standing wave outside the inner boundary layer. Figure 3 shows the amplitude $G(r)$ and phase $\beta(r)$ of the azimuthal vorticity (2.18), which also differ significantly between the open and enclosed flows. In the open flow the vorticity amplitude is almost constant for $\omega \sim 1$, and decays exponentially for $\omega \gg 1$ while the phase is linear in $r$, corresponding to a wave of azimuthal vorticity propagating from 
the inner to the outer cylinder. In the enclosed flow, the azimuthal vorticity amplitude is very small in the bulk of the return flow, reflecting the fact that the axial velocity is almost constant, except in a boundary layer of significant vorticity on the outer cylinder. The plot of the vorticity phase shows that in the enclosed flow waves of azimuthal vorticity propagate into the interior from each of the cylinder walls. They meet in the return flow region, where the amplitude of the azimuthal vorticity is at a minimum. The return flow and the associated waves of azimuthal vorticity are global and significant consequences of the endwall effects that are manifested throughout the whole flow.

\subsection{Asymptotic expressions for the open flow}

In order to compare with the solutions of the basic flow obtained by $\mathrm{Hu} \&$ Kelly (1995) in the narrow gap case for the open flow, we need asymptotic expressions for $f$ and $g$. The asymptotic expansions of $I_{n}(y), K_{n}(y)$, from Abramowitz \& Stegun (1972), are

$$
\begin{aligned}
& I_{n}(y) \sim \frac{\mathrm{e}^{y}}{(2 \pi y)^{1 / 2}}\left\{1-\frac{4 n^{2}-1}{8 y}+\ldots\right\} \quad(|\arg y|<\pi / 2), \\
& K_{n}(y) \sim\left(\frac{\pi}{2 y}\right)^{1 / 2} \mathrm{e}^{-y}\left\{1+\frac{4 n^{2}-1}{8 y}+\ldots\right\} \quad(|\arg y|<3 \pi / 2),
\end{aligned}
$$

valid for $|y|$ large, i.e. $\omega^{1 / 2} r_{i} \gg 1$; the conditions on the argument of $y$ are clearly satisfied because $\arg y=\pi / 4$. Taking the first term in these expansions, we obtain for $f$ and $g$

$$
\begin{aligned}
& f(r) \sim\left(\frac{r_{i}}{r}\right)^{1 / 2} \frac{\sinh \left(\omega^{1 / 2}\left(r_{o}-r\right) \mathrm{e}^{\mathrm{i} \pi / 4}\right)}{\sinh \left(\omega^{1 / 2} \mathrm{e}^{\mathrm{i} \pi / 4}\right)} \\
& g(r) \sim\left(\frac{\omega r_{i}}{r}\right)^{1 / 2} \frac{\cosh \left(\omega^{1 / 2}\left(r_{o}-r\right) \mathrm{e}^{\mathrm{i} \pi / 4}\right)}{\sinh \left(\omega^{1 / 2} \mathrm{e}^{\mathrm{i} \pi / 4}\right)} \mathrm{e}^{\mathrm{i} \pi / 4} .
\end{aligned}
$$

Since $\omega^{1 / 2} r_{i}=\omega^{1 / 2} e /(1-e)$, the above expressions are valid not only in the narrow gap case $e \rightarrow 1$, but also for finite gap values provided that $\omega^{1 / 2} \gg 1$. In Hu \& Kelly (1995) an explicit expression for $f$ is obtained in the narrow gap limit case $(e \rightarrow 1)$; our expression (2.30) is the same, except for the term $\left(r_{i} / r\right)^{1 / 2}$, which in the narrow gap case is taken as 1 .

The expression (2.30) exactly satisfies the boundary conditions; both (2.30) and (2.31) are very good approximations for $w$ and $\eta$ not only in the asymptotic case but also for $\omega^{1 / 2} r_{i} \approx 1$, as can be seen in figure 4. Expression (2.31) is minus the derivative of (2.30), treating the factor $\left(r_{i} / r\right)^{1 / 2}$ as constant, which is consistent with the asymptotic approximation. However, for $\omega^{1 / 2} r_{i} \approx 1$ it is necessary to treat this factor as variable, which following differentiation of (2.30) introduces an additional term $-0.5 f / r$ to (2.31). This then gives a good approximation for $\eta$ even when $\omega^{1 / 2} r_{i} \approx 1$ (see figure 4 ).

When $\omega^{1 / 2} \gg 1$ we get very simple expressions for the modulus and phase of $w$ and $\eta$ :

$$
\begin{array}{ll}
W(r) \sim\left(\frac{r_{i}}{r}\right)^{1 / 2} \mathrm{e}^{-(\omega / 2)^{1 / 2}\left(r-r_{i}\right)}, & \alpha(r) \sim-\left(\frac{\omega}{2}\right)^{1 / 2}\left(r-r_{i}\right), \\
G(r) \sim\left(\frac{\omega r_{i}}{r}\right)^{1 / 2} \mathrm{e}^{-(\omega / 2)^{1 / 2}\left(r-r_{i}\right)}, & \beta(r) \sim \frac{\pi}{4}-\left(\frac{\omega}{2}\right)^{1 / 2}\left(r-r_{i}\right),
\end{array}
$$

valid throughout the gap, with the exception of a small region near the outer cylinder, 

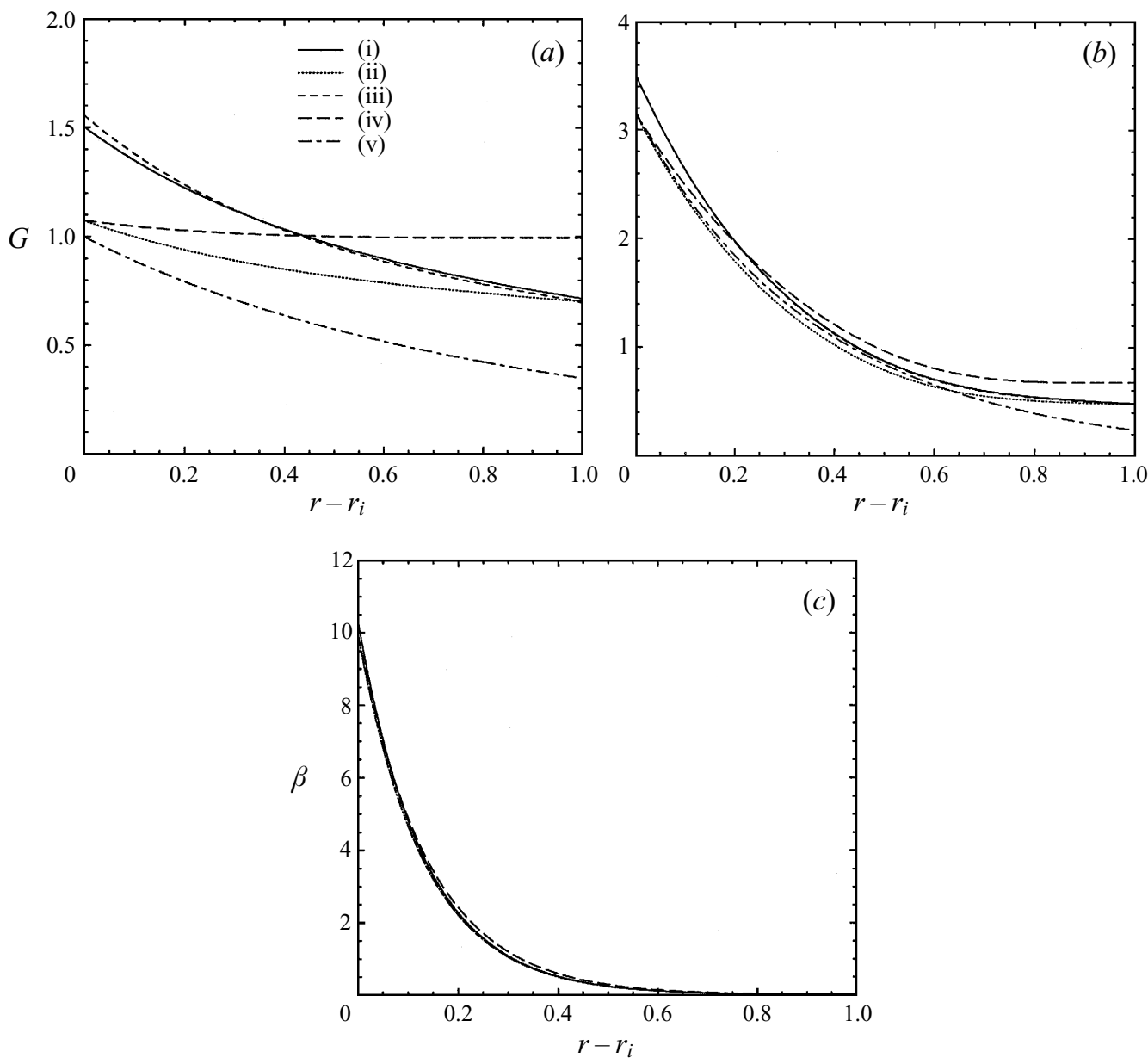

FIGURE 4. Comparisons of the azimuthal vorticity amplitude profiles for gap ratio $e=0.5$ between (i) the exact solution (2.19); (ii) the asymptotic solution for $\omega^{1 / 2} r_{i} \gg 1$ (2.31); (iii) the asymptotic solution (2.31) corrected with the $-0.5 f / r$ term; (iv) the narrow gap approximation, $\left(r_{i} / r\right)^{1 / 2} \rightarrow 1$; and (v) the asymptotic solution for $\omega^{1 / 2} \gg 1(2.33)$, for the open flow case with $(a) \omega=10^{0},(b)$ $\omega=10^{1}$, and $(c) \omega=10^{2}$.

of width $(2 / \omega)^{1 / 2}$ known as the Stokes length, as can be seen in figure 4 . All the comparisons between the exact solution and the various asymptotic approximations shown in figure 4 are for a wide gap $e=0.5$. Comparisons at $e \sim 0.9$ show very little difference, indicating that a narrow gap approximation is appropriate for a representation of the basic flow in the experiments of Weisberg et al. (1997).

Expressions (2.32), (2.33) show that the basic flow consists of the basic Couette flow solution (2.13) with a superimposed oscillating axial velocity. Waves of azimuthal vorticity propagate throughout the fluid from the inner cylinder to the outer one. The moduli of these $w$ and $\eta$ waves decrease exponentially and their phases vary linearly with $r$. The oscillatory part is $\sin \left(\omega t-(\omega / 2)^{1 / 2} r+\right.$ constant $)$, which gives a radial wavenumber equal to $(\omega / 2)^{1 / 2}$. This dispersion relation gives a group velocity twice the phase velocity $(2 \omega)^{1 / 2}$. The number of complete oscillations of both $w$ and $\eta$ in the gap is given by $N_{o s c}=(\omega / 2)^{1 / 2} /(2 \pi)$; therefore in the $\omega \gg 1$ case we have a large number of complete periods. However, the damping factor in the gap is 
$\exp \left((\omega / 2)^{1 / 2} r\right)$, and so only one or two complete oscillations (near the inner cylinder) are significant.

In order to understand the phase adjustment near the outer cylinder it is worth analysing the special case $r_{o} \rightarrow \infty$. This case corresponds to a cylinder rotating and oscillating axially inside a fluid in a very large container. The previous analysis is valid with only small changes. Now there is only one length scale, the inner radius $r_{i}$. The governing equations (2.1) remain the same, with $r_{i}$ instead of $d$ used as the length scale. However, the boundary conditions change to

$$
u(1)=0, \quad v(1)=R e_{i}, \quad w(1, t)=R e_{a} \sin \omega t, \quad v(r \rightarrow \infty) \rightarrow 0,
$$

and the solution for the basic flow is simpler:

$$
u=0, v=R e_{i} / r, w=R e_{a} \operatorname{Im}\left(f(r) \mathrm{e}^{\mathrm{i} \omega t}\right), \eta=R e_{a} \operatorname{Im}\left(g(r) \mathrm{e}^{\mathrm{i} \omega t}\right),
$$

where now

$$
f(r)=K_{0}(y) / K_{0}\left(y_{i}\right), \quad g(r)=\omega^{1 / 2} \mathrm{e}^{\mathrm{i} \pi / 4} K_{1}(y) / K_{0}\left(y_{i}\right) .
$$

The asymptotic expressions (2.36) for $\omega^{1 / 2} \gg 1$ are now precisely (2.32)-(2.33). When the outer cylinder is absent, there is no phase adjustment in order to satisfy the outer boundary conditions, as expected. We now have a simple $r^{-1}$ vortex generated by the rotation of the cylinder at $r_{i}=1$ with a superimposed oscillating axial velocity and damped waves of azimuthal vorticity propagating throughout the fluid.

\section{Floquet analysis}

In the preceding Section the basic flow was obtained. We now perturb this basic state by a small disturbance which is assumed to vary periodically in the azimuthal and axial directions:

$$
\begin{aligned}
& \boldsymbol{v}(r, \theta, z, t)=\boldsymbol{v}_{B}(r, t)+\mathrm{e}^{\mathrm{i}(n \theta+k z)} \boldsymbol{u}(r, t), \\
& p(r, \theta, z, t)=p_{B}(r, z, t)+p^{\prime}(r, t) \mathrm{e}^{\mathrm{i}(n \theta+k z),},
\end{aligned}
$$

where $\boldsymbol{v}_{B}=\left(0, v_{B}, w_{B}\right)$ is given by (2.13) and (2.14) and the boundary conditions for $\boldsymbol{u}$ are homogeneous, $\boldsymbol{u}\left(r_{i}\right)=\boldsymbol{u}\left(r_{o}\right)=\mathbf{0}$. In the enclosed case, one may wonder if a disturbance to the overall axial pressure gradient must also be added. A straightforward calculation using the incompressibility condition and boundary conditions for $v$ shows that the perturbed velocity field has a zero axial mass flow at any instant in time, and therefore the basic flow solution $p_{2}$ does not change. Linearizing the Navier-Stokes equations about the basic solution, we obtain

$$
\partial_{t} \boldsymbol{u}=-\nabla p^{\prime}+\Delta \boldsymbol{u}-\boldsymbol{v}_{B} \cdot \nabla \boldsymbol{u}-\boldsymbol{u} \cdot \nabla \boldsymbol{v}_{B} .
$$

The spatial discretization of the problem, in order to solve (3.3) numerically, is accomplished by projecting (3.3) onto a suitable basis. The space of divergence-free vector fields satisfying the boundary conditions of the problem is

$$
V=\left\{\boldsymbol{u} \in\left(\mathscr{L}_{2}\left(r_{i}, r_{o}\right)\right)^{3} \mid \nabla \cdot \boldsymbol{u}=0, \boldsymbol{u}\left(r_{i}\right)=\boldsymbol{u}\left(r_{o}\right)=0\right\},
$$

where $\left(\mathscr{L}_{2}\left(r_{i}, r_{o}\right)\right)^{3}$ is the Hilbert space of square-integrable vectorial functions defined in the interval $\left(r_{i}, r_{o}\right)$, with the inner product

$$
\langle\boldsymbol{u}, \boldsymbol{v}\rangle=\int_{r_{i}}^{r_{o}} \boldsymbol{u}^{*} \cdot \boldsymbol{v} r \mathrm{~d} r
$$


where * denotes the complex conjugate. For any $\boldsymbol{u} \in V$ and any function $p$, we have $\langle\boldsymbol{u}, \nabla p\rangle=0$. Therefore expanding $\boldsymbol{u}$ in a suitable basis of $V$ :

$$
\boldsymbol{u}=\sum_{\alpha} a_{\alpha} \boldsymbol{u}_{\alpha}, \quad \boldsymbol{u}_{\alpha} \in V
$$

and projecting the linearized equations (3.3) onto $V$ the pressure term disappears, and we get a system of ordinary differential equations for the coefficients $a_{\alpha}$ :

$$
\sum_{\beta}\left\langle\tilde{\boldsymbol{u}}_{\alpha}, \boldsymbol{u}_{\beta}\right\rangle \dot{a}_{\beta}=\sum_{\beta}\left\langle\tilde{\boldsymbol{u}}_{\alpha}, \Delta \boldsymbol{u}_{\beta}-\boldsymbol{v}_{B} \cdot \nabla \boldsymbol{u}_{\beta}-\boldsymbol{u}_{\beta} \cdot \nabla \boldsymbol{v}_{B}\right\rangle a_{\beta} .
$$

We implement a Petrov-Galerkin scheme, where the basis used to expand the unknown velocity, $\left\{\boldsymbol{u}_{\alpha}\right\}$, differs from that used to project the equations, $\left\{\tilde{\boldsymbol{u}}_{\alpha}\right\}$. A comprehensive analysis of the method can be found in Moser, Moin \& Leonard (1983) or Canuto et al. (1988). The divergence-free condition for a velocity field of the form (3.1) is $\mathrm{D}_{+} u+\mathrm{i} n v / r+\mathrm{i} k w=0$, and a basis for $V$ is obtained by taking

$$
\begin{aligned}
& \boldsymbol{u}_{2 j-1}=\left(f_{j}, 0, \mathrm{iD}_{+} f_{j} / k\right), \\
& \boldsymbol{u}_{2 j}=\left(0, g_{j},-n g_{j} /(k r)\right),
\end{aligned}
$$

where $f_{j}$ and $g_{j}$ must satisfy the homogeneous boundary conditions $f_{j}=f_{j}^{\prime}=g_{j}=0$ on $r_{i}$ and $r_{o}$. The vorticity vectors associated with this basis are

$$
\begin{aligned}
& \nabla \times \boldsymbol{u}_{2 j-1}=\left(-n \mathrm{D}_{+} f_{j} /(k r),-\mathrm{i}\left(\mathrm{DD}_{+}-k^{2}\right) f_{j} / k,-\mathrm{i} n f_{j} / r\right), \\
& \nabla \times \boldsymbol{u}_{2 j}=\left(-\mathrm{i}\left(k^{2}+n^{2} / r^{2}\right) g_{j} / k, n \mathrm{D}\left(g_{j} / r\right) / k, \mathrm{D}_{+} g_{j}\right) .
\end{aligned}
$$

In the axisymmetric case, $n=0$ and the $f_{j}$ construct the Stokes streamfunction

$$
\psi=r \sum_{j=1}^{M} a_{2 j-1} f_{j}(r) \mathrm{e}^{\mathrm{i} k z},
$$

describing the toroidal (meridional) components of the flow and the $g_{j}$ construct the angular momentum

$$
\Gamma=r v=r \sum_{j=1}^{M} a_{2 j} g_{j}(r) \mathrm{e}^{\mathrm{i} k z},
$$

describing the poloidal (azimuthal) components of the flow.

Introducing the new radial coordinate $x=2\left(r-r_{i}\right)-1, x \in[-1,+1]$ and using Chebyshev polynomials $T_{j}$, a simple choice for $f_{j}$ and $g_{j}$, which satisfies the homogeneous boundary conditions, is

$$
f_{j}(r)=\left(1-x^{2}\right)^{2} T_{j-1}(x), \quad g_{j}(r)=\left(1-x^{2}\right) T_{j-1}(x),
$$

where $j$ ranges from 1 to $M$, the number of Chebyshev polynomials used. In order to preserve the orthogonality relationships between the Chebyshev polynomials, and to avoid $1 / r$ factors in the inner products in (3.7), a suitable choice for the projection basis $\tilde{\boldsymbol{u}}$ is

$$
\tilde{f}_{j}(r)=r^{3}\left(1-x^{2}\right)^{3 / 2} T_{j-1}(x), \quad \tilde{g}_{j}(r)=r^{3}\left(1-x^{2}\right)^{1 / 2} T_{j-1}(x) .
$$

With this choice, all the inner products in (3.7) involve polynomials, except those containing the axial velocity $w_{B}$, and therefore can be numerically computed exactly using Gauss-Chebyshev quadrature (Isaacson \& Keller 1966). Finally we obtain a 
system of the form

$$
\boldsymbol{G} \dot{\boldsymbol{x}}=\boldsymbol{H}(t) \boldsymbol{x}=(\boldsymbol{A}+\boldsymbol{B} \sin \omega t+\boldsymbol{C} \cos \omega t) \boldsymbol{x},
$$

where the vector $\boldsymbol{x}$ contains the real and imaginary parts of the coefficients $a_{\alpha}$ in (3.6), and the matrices $\boldsymbol{A}, \boldsymbol{B}, \boldsymbol{C}, \boldsymbol{G}$ are time independent, with $\boldsymbol{G}$ positive definite; details of the explicit expressions for $\boldsymbol{G}$ and $\boldsymbol{H}$ are given in the Appendix.

The stability analysis of the basic flow is reduced to the determination of the growth rates of solutions of the linear system with periodic coefficients (3.16). This is accomplished using classical Floquet theory (e.g. Joseph 1976; Guckenheimer \& Holmes 1986) and numerical integration. The fundamental matrix of (3.16) is the solution of the system

$$
\boldsymbol{G} \dot{\boldsymbol{X}}=\boldsymbol{H}(t) \boldsymbol{X}, \quad \boldsymbol{X}(0)=\boldsymbol{I},
$$

where $\boldsymbol{I}$ is the identity matrix. Integrating over a complete period $T=2 \pi / \omega$, we obtain the monodromy matrix of the system $\boldsymbol{X}(T)$, whose eigenvalues $\gamma_{j}, j=1, \ldots, 4 M$, called Floquet multipliers, control the growth rate of the perturbations. If $\left|\gamma_{j}\right|<1$ for all $j$, all the perturbations decrease after a complete period of the oscillation and the basic flow is stable. If there exists a $j$-value such that $\left|\gamma_{j}\right|>1$, then perturbations of the corresponding mode grow after a complete period and the basic flow is unstable. When an eigenvalue crosses the unit circle $\left|\gamma_{j}\right|=1$, the basic flow undergoes a bifurcation. The kind of bifurcation depends on the critical eigenvalue $\gamma_{j}$. If its value is +1 , we have a saddle-node bifurcation or a pitchfork bifurcation depending on the symmetries of the system; if it is -1 , we have a period-doubling bifurcation, and if we have a pair of complex-conjugate eigenvalues of moduli 1 , the bifurcating solution is quasi-periodic (a kind of Hopf bifurcation for periodic orbits; see Guckenheimer \& Holmes 1986 for details and examples).

The equations and boundary conditions of the problem are invariant to axial translations and azimuthal rotations. The time-dependent axial oscillations of the inner cylinder break the reflection symmetry in the axial direction and the invariance to translations in time. However, due to the harmonic character of the axial oscillations, the system is invariant under the symmetry operation

$$
z \rightarrow-z, \quad w \rightarrow-w, \quad t \rightarrow t+\pi / \omega .
$$

Hence two solutions related by this symmetry with $(n, k)$ and $(n,-k)$ are essentially the same. Also, the complex conjugation of a $(n, k)$ perturbation (3.1) gives another solution with $(-n,-k)$. Therefore, one need only to consider $n \geqslant 0, k \geqslant 0$.

Our numerical technique consists of an integration scheme, an eigenvalue finder, and a continuation procedure in order to obtain curves of critical values of $R e_{i}$ and $k$ as functions of the system parameters.

The temporal integration of the initial value problem (3.17) over the interval $t \in[0, T]$ is performed by a second-order implicit method known as the trapezoidal rule; see Hairer \& Wanner (1991) for details concerning its theoretical stability and convergence properties. Systems of the form (3.17) may exhibit characteristics of stiff ordinary differential equations. Our solutions obtained by the trapezoidal rule have been checked for small values of $\omega$ and large values of $R e_{a}$, the frequency and amplitude of the axial motion respectively, using a fourth-order stiffly accurate method known as Radau IIA (Hairer \& Wanner 1991) and the two results differ by less than one part in ten thousand. A uniform time step dependent upon $R e_{a}$, $\omega$, and the number of radial modes $M$ was used. For $M=10$, we determined that the critical $R e_{i}$ and $k$ could be determined to one part in ten thousand by using 
(a)

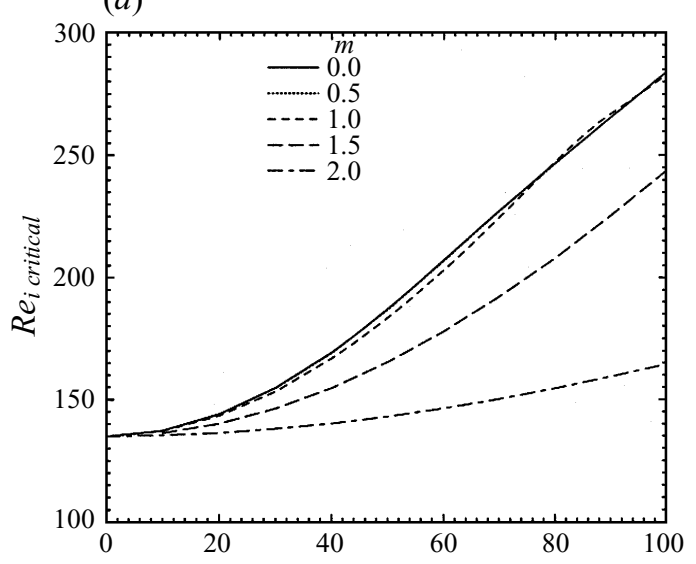

(b)
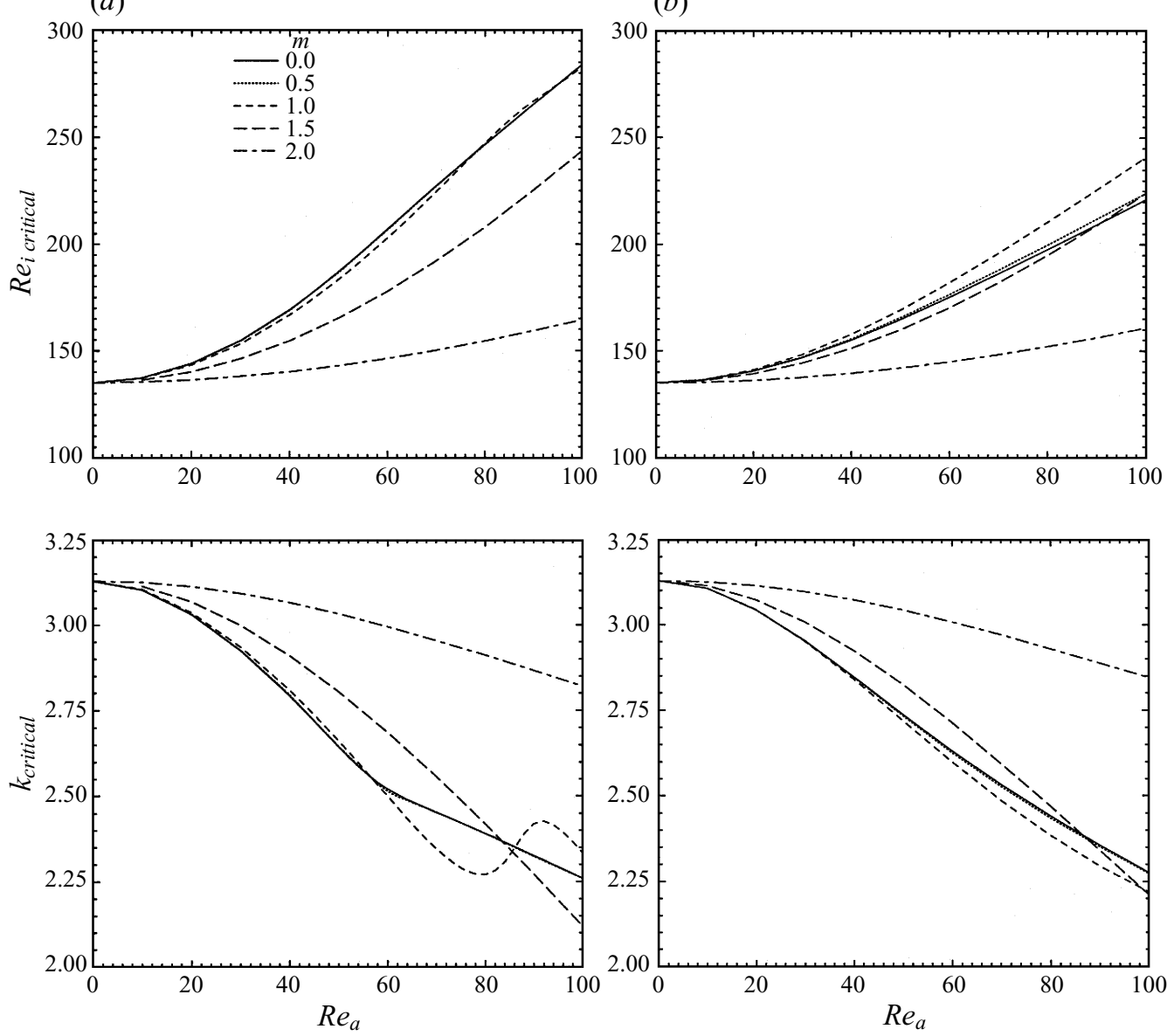

FIGURE 5. Critical $R e_{i}$ and $k$ versus $R e_{a}$ in $(a)$ the enclosed flow and $(b)$ the open flow for $e=0.905, n=0$, and various $\omega=10^{m}, m$ as indicated.

$\max \left(40 \max \left(R e_{a}, 20\right) / \omega, 200\right)$ time steps in one period over the range of $R e_{a}$ and $\omega$ considered.

\section{Results of the Floquet analysis}

To begin with, we shall restrict our attention to parameter regimes investigated experimentally by Weisberg et al. (1997) and theoretically by Hu \& Kelly (1995). Specifically, in this present study we shall only consider flows with the outer cylinder stationary, i.e. $R e_{o}=0$, and a radius ratio $e=0.905$ corresponding to Weisberg et al.'s experimental apparatus. This reduces the problem to one with two externally imposed parameters representing the amplitude and frequency of the periodic forcing, $R e_{a}$ and $\omega$ respectively. The object is to determine the critical $R e_{i}$, corresponding to the lowest rotation rate of the inner cylinder, for a given $R e_{a}$ and $\omega$, at which Taylor vortex flow does not decay for long times, and the corresponding critical axial and azimuthal wavenumbers, $k$ and $n$.

The main results are presented in figures 5 and 6 , giving the critical $R e_{i}$ and $k$ versus $R e_{a}$ and versus $\omega$ respectively, for $n=0$, in both the enclosed and open 
(a)
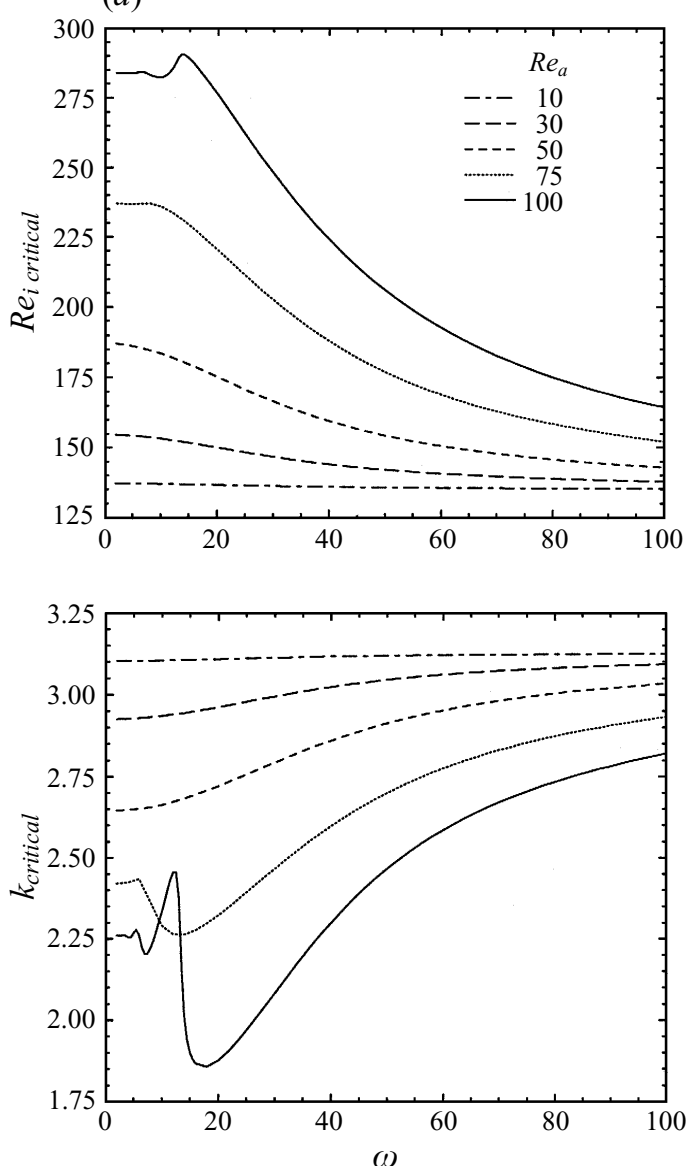

(b)

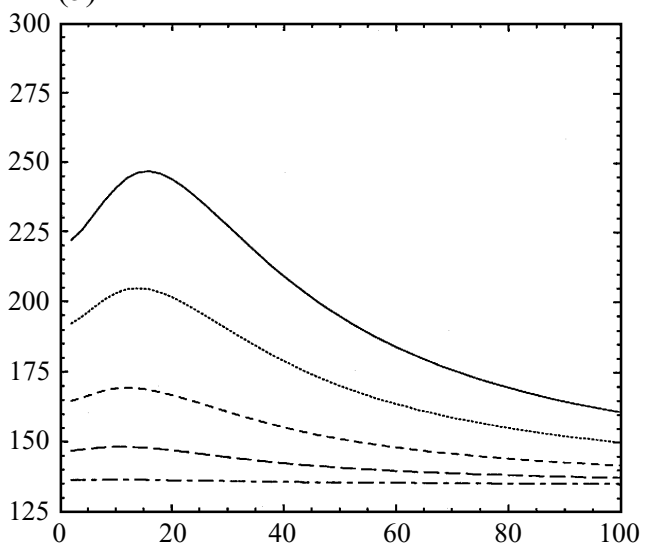

Figure 6. Critical $R e_{i}$ and $k$ versus $\omega$ in $(a)$ the enclosed flow and $(b)$ the open flow for $e=0.905$, $n=0$, and various $R e_{a}$ as indicated.

flows. For the parameter ranges considered, we have found that $n=0$ is the critical azimuthal mode, in accord with the narrow gap analysis of Hu \& Kelly (1995) and the experiments of Weisberg et al. (1997), except in some narrow windows of parameter space corresponding to small $\omega$ and large $R e_{a}$ (see below). When instability sets in via an axisymmetric mode, $n=0$, the new state is synchronous with the basic state, i.e. no new frequency is introduced by the bifurcation. Figures 5 and 6 show that the degree of stabilization, i.e. the relative difference between critical $R e_{i}$ for a given $R e_{a}$ and $\omega$ and the critical $R e_{i}$ for $R e_{a}=0$, is always greater for the enclosed flow. The critical wavenumber decreases more rapidly for increasing $R e_{a}$ and decreasing $\omega$ in the enclosed flow than in the open flow. The flow under consideration represents a competition between annular Stokes flow and circular Couette flow. When $R_{a}$ is increased, the relative importance of the Stokes flow component is enhanced. When $\omega$ is reduced the influence of the Stokes flow penetrates deeper into the interior (an increase in the Stokes length $\left.(2 / \omega)^{1 / 2}\right)$ and so also represents a relative increase in the influence of the Stokes flow component. The annular Stokes flow in the absence of the circular Couette component is very stable, i.e. the flow is independent of $z$ for a very large range of $R e_{a}$ and $\omega$. In the range of $R e_{a}$ and $\omega$ considered in this 

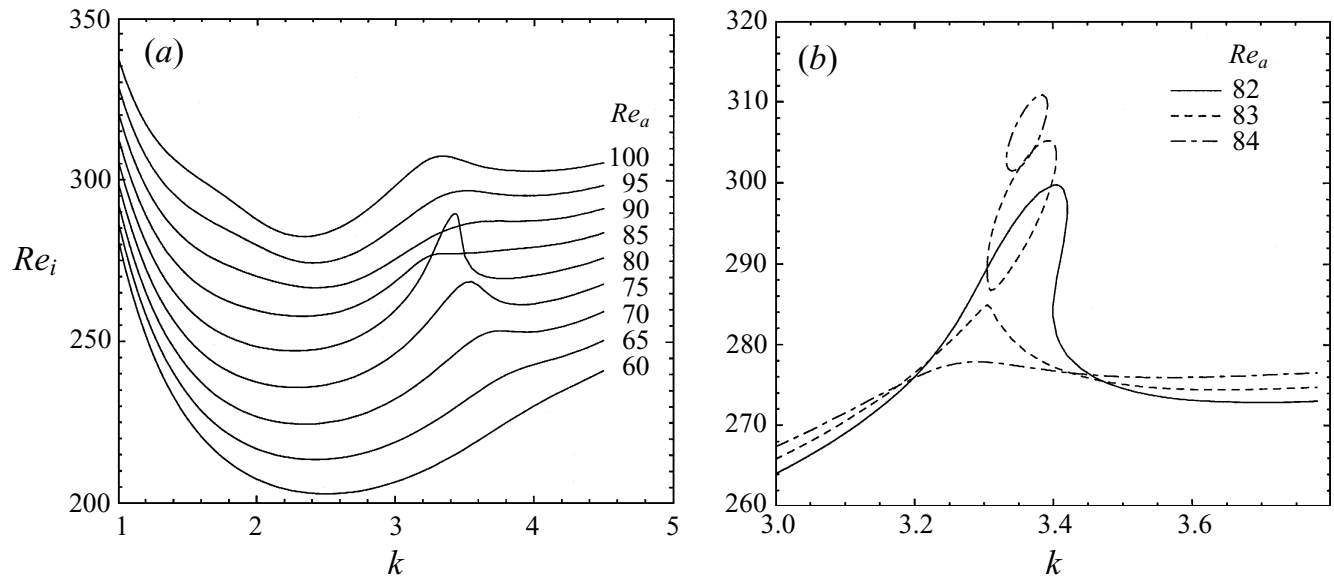

FiguRE 7. Stability boundaries in $\left(R e_{i}, k\right)$-space for the enclosed flow with $e=0.905, n=0, \omega=10$, and $R e_{a}$ as indicated. (b) Detail of $(a)$.
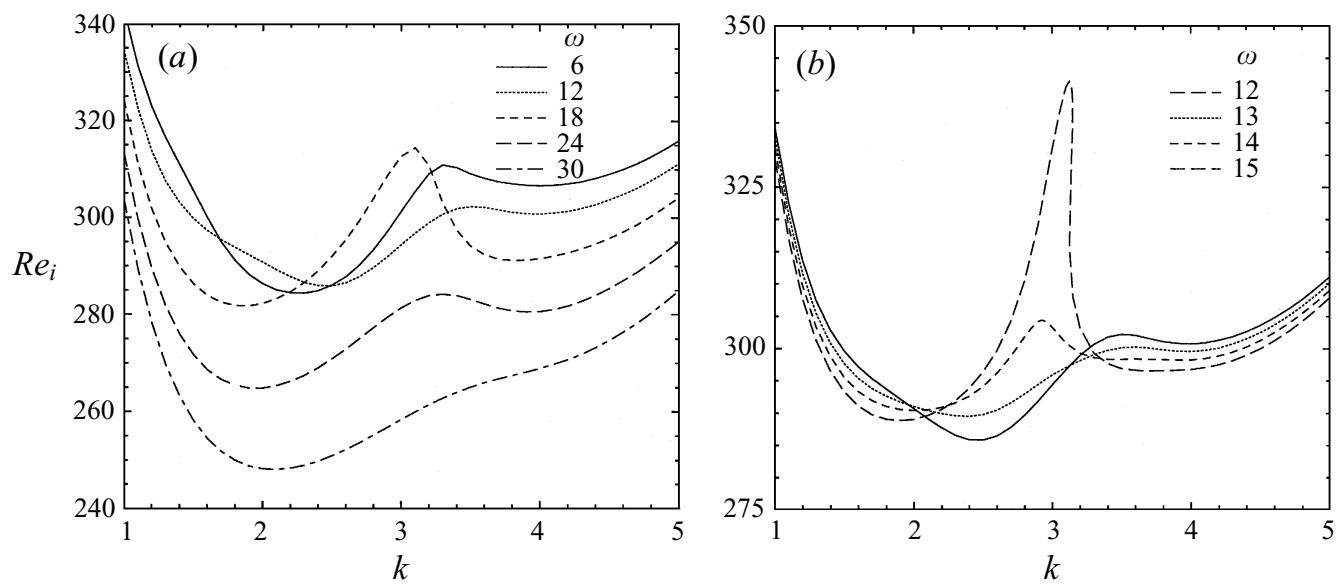

FIGURE 8. Stability boundaries in $\left(R e_{i}, k\right)$-space for the enclosed flow with $e=0.905, n=0$, $R e_{a}=100$, and $\omega$ as indicated. (b) Detail of $(a)$

study, we have verified numerically that annular Stokes flow (i.e. the present flow with $R e_{i}=0$ ) is stable for all $k$ and $n$ considered; von Kerczek \& Davis (1974) have also demonstrated stability over a large region of parameter space for finite planar Stokes flow. For the Stokes component in the present flow, the least stable of all the (stable) wavenumbers is at $k=0$. The circular Couette flow, in the absence of axial oscillations, has a preferred axial wavenumber for the onset of Taylor vortex flow $k \approx \pi$. As the Stokes component of the flow becomes dominant, we observe a shift from $k \approx \pi$ to smaller wavenumbers for the oscillatory Taylor-Couette flow, as illustrated in figures 5 and 6.

The enclosed flow shown in figures 5 and 6 behaves in a peculiar fashion at large $R e_{a}$ and small $\omega$, as manifested predominantly in the variations of the critical $k$ as $R e_{a}$ increases and $\omega$ decreases, where we see that there is a rapid increase in the critical $k$ superimposed on the general trend of decreasing $k$. This type of behaviour is indicative of modal interactions, and we investigate this by plotting stability boundary curves of $R e_{i}$ at onset of sustained Taylor vortex flow versus $k$ for $R e_{a}$ and $\omega$ in the 


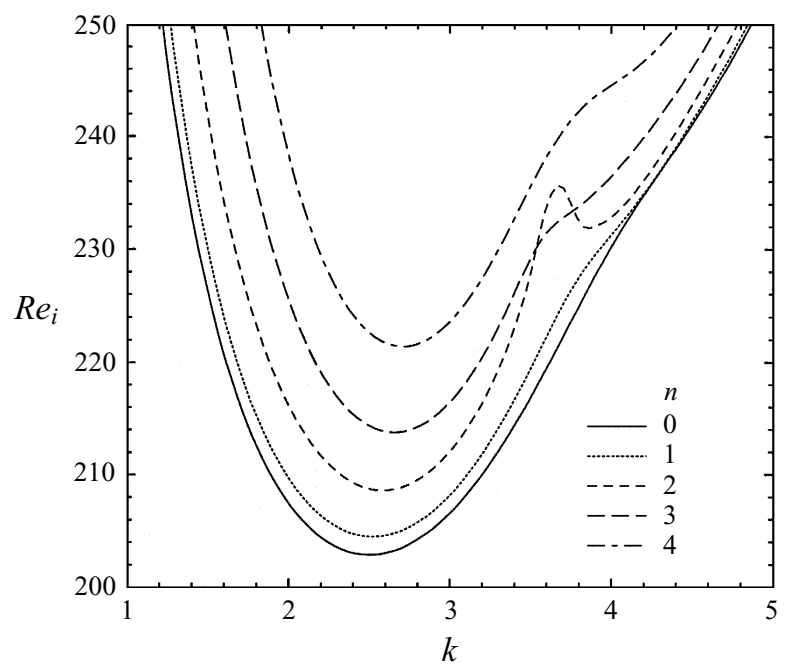

FIGURE 9. Stability boundaries in $\left(R e_{i}, k\right)$-space for the enclosed flow with $e=0.905, \omega=10$, $R e_{a}=60$, and $n$ as indicated.

range where this modal interaction is apparent. Figure 7 gives these curves for $\omega=10$ and $60 \leqslant R e_{a} \leqslant 100$ corresponding to the rapid change in $k$ indicated in figure 5 . These clearly show that this peculiar behaviour is associated with the development of a second local minimum in the critical $R e_{i}$ versus $k$ curves. The new local minimum develops with increasing $R e_{a}$ as an inflection point on the curve appears at $R e_{a} \approx 65$ and $k \approx 4$. The slope of the curve at the inflection point is positive and decreases with increasing $R e_{a}$. When the slope at the inflection point becomes negative, a new local minimum at a $k$ larger than that corresponding to the inflection point appears. As the slope continues to become more negative, the local minimum shifts to lower $k$, and at $R e_{a} \approx 83$ the $R e_{i}$ versus $k$ curve pinches off forming an isolated island of stable flow (stable to axisymmetric perturbations), as shown in the detail of figure 7(b). This island decreases in size rapidly with increasing $R e_{a}$. During the pinch-off phase, the local minimum and the global minimum are drawn closer together, hence the S-bend in the critical $k$ (global minimum) curve in figure 5. The local minimum does not becomes the global minimum (in this parameter regime), but it is extremely influential in bringing the critical $k$, corresponding to the global minimum, back closer to $\pi$. Similar behaviour is observed in figure 8 giving the curves of critical $R e_{i}$ versus $k$ for $R e_{a}=100$ and $6 \leqslant \omega \leqslant 30$ corresponding to the rapid change in $k$ indicated in figure 6 . Note that these modal interactions were not observed in the open flow case over the same range of $R e_{a}$ and $\omega$.

Although the $n \neq 0$ azimuthal modes are not dominant over most of the parameter range investigated, similar modal interactions and pinch-offs occur for $n \neq 0$ as illustrated in figure 9, which shows critical $R e_{i}$ versus $k$ curves for $n=0,1,2,3$, and $4, \omega=10$, and $R e_{a}=60$. In the narrow windows of parameter space where the pinch-offs occur, we have found that the $n \neq 0$ modes are the most dangerous. The pinch-offs have been found to occur in the regions of parameter space where the critical $R e_{i}$ versus $\omega$ or $R e_{a}$ curves appear peculiar. These occur for $R e_{a}>70$, and a narrow wedge in $\omega \sim O(10)$. Figure 10 reproduces the critical $R e_{i}$ and $k$ curves in figure 6 for $R e_{a}=75$ and 100 . For $R e_{a}=75$ and $5.5 \leqslant \omega \lesssim 10$ the $n=1$ azimuthal mode is dominant, and for $R e_{a}=100$ and $11.8 \leqslant \omega \lesssim 17.8$ the $n=2$ azimuthal mode is 
(a)
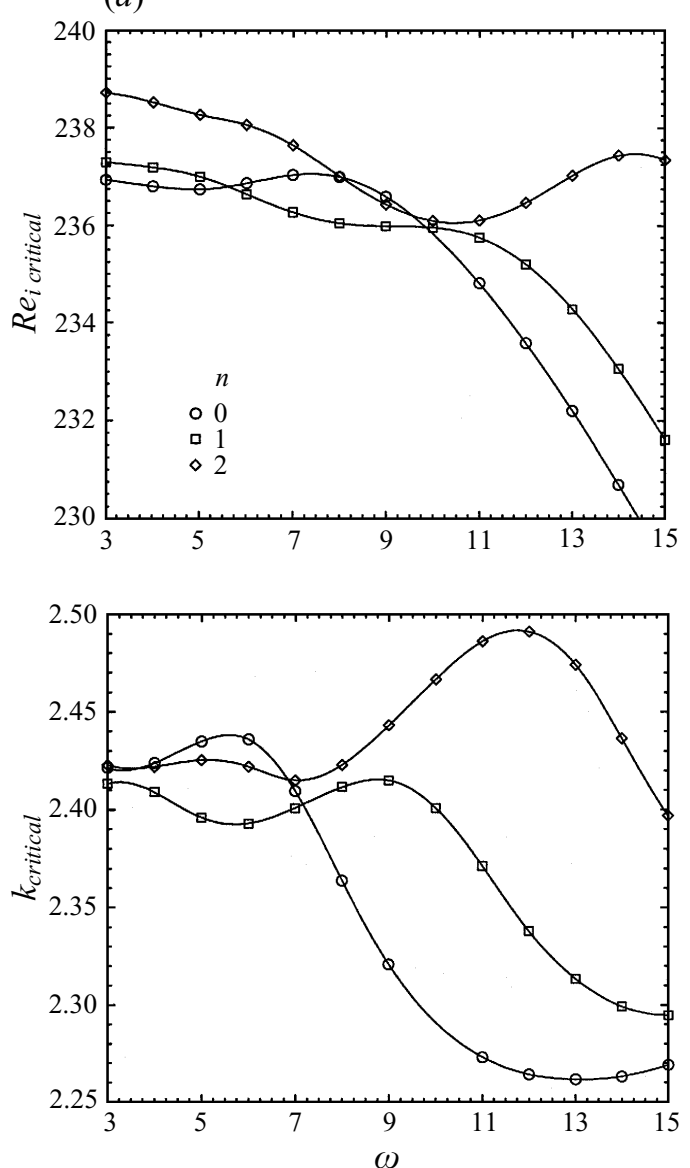

(b)
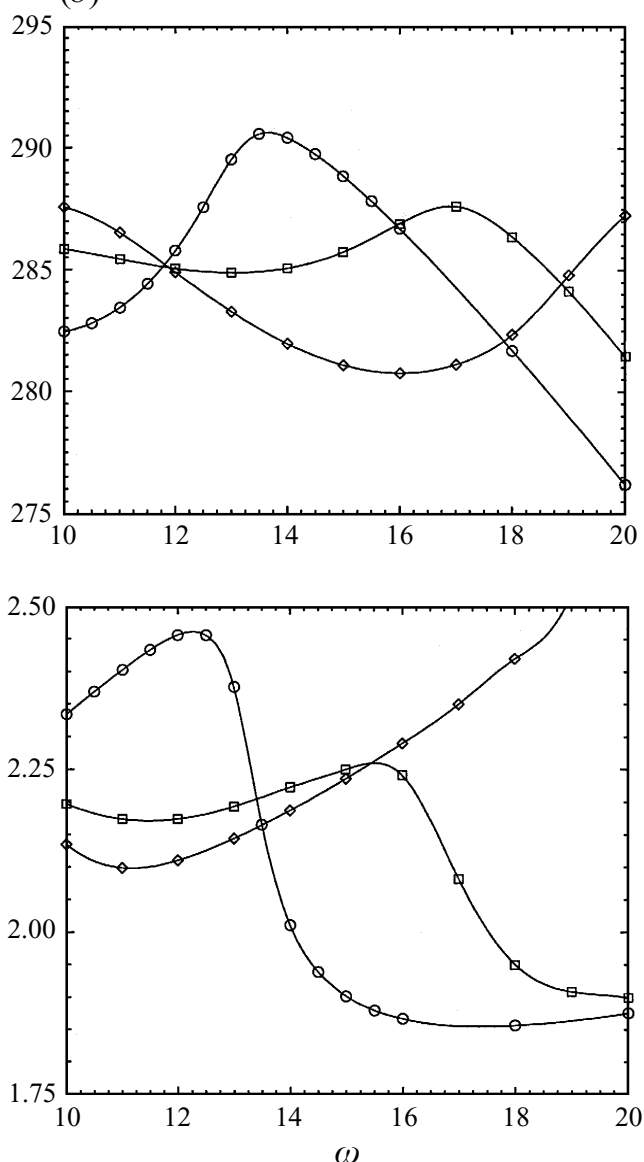

Figure 10. Critical $R e_{i}$ and $k$ versus $\omega$ in the enclosed flow with $e=0.905$ for $(a) R e_{a}=75$ and $(b)$ $R e_{a}=100, e=0.905$, and various azimuthal modes $n$ as indicated.

dominant. At the points where the azimuthal mode switching occurs, the wavelength of the dominant mode undergoes a discontinuous jump. For $n \neq 0$ dominant modes, the bifurcation is to a quasi-periodic state consisting of the frequency of the basic flow, $\omega$, and a new frequency $\omega_{1}$ or $\omega_{2}$, corresponding to either $n=1$ or $n=2$ respectively. This is the Hopf bifurcation for periodic orbits mentioned in $\$ 3$. For the parameter ranges considered, these new frequencies varied with $\operatorname{Re}_{a}$ and $\omega$. For $R e_{a}=75$ and $5.5 \lesssim \omega \lesssim 10, \omega_{1} \approx 14$, and for $R e_{a}=100$ and $11.8 \lesssim \omega \lesssim 17.8, \omega_{2} \approx 35$.

\subsection{Comparison with experiments}

The only experiments on Taylor-Couette flow with axial oscillations of the inner cylinder known to us are those of Weisberg et al. (1997). The geometry of the experimental apparatus consisted of a radius ratio $e=0.905$ and aspect ratio $\Lambda=$ length of cylinders $/ d=150$, and had stationary rigid endwalls at both ends of the cylinders. The experiments were all conducted with a stationary outer cylinder $\left(R e_{o}=0\right)$. Owing to mechanical constraints, the two driving parameters $R e_{a}$ and $\omega$ were coupled by a linear relationship $R e_{a}=C \omega$, but the constant $C$ could be varied 

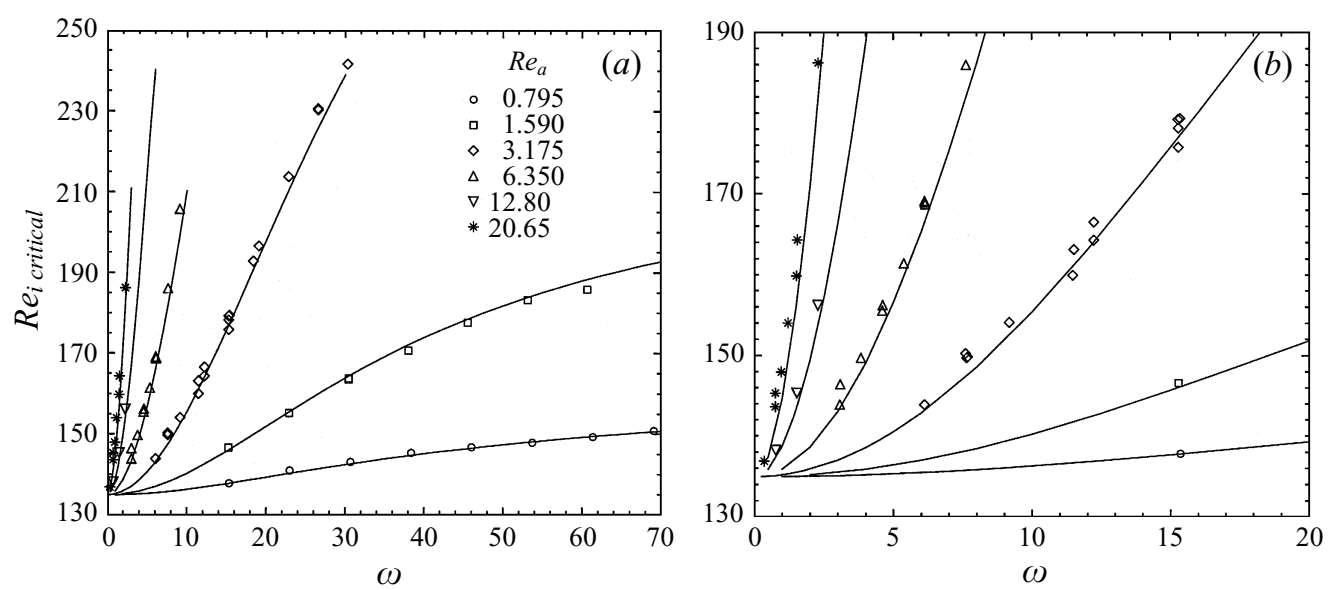

FiguRE 11. Critical $R e_{i}$ versus $\omega$ for various $R e_{a}$, defined by the relationship $R e_{a}=C \omega$ with $C$ as indicated, compared with the corresponding experimental data of Weisberg et al. (1997) (symbols). (b) Detail of $(a)$.

in different sequences of experiments, thus making a large range of parameter space accessible.

In comparing our Floquet analysis to the experiments, we make some idealizations. Specifically, we idealize the finite aspect ratio $\Lambda=150$ as $\Lambda=\infty$ and we only include the global effects of the endwalls via a time-periodic but spatially constant pressure gradient term, i.e. the enclosed flow formulation. We do however include fully the curvature effects, using $e=0.905$. All of Weisberg et al.'s data for the critical value of $R e_{i}$ are presented in figure 11 by open symbols, each different symbol corresponding to different constants $C$ as indicated. The solid lines are our Floquet analysis results. It is clear that the agreement is uniformly to within $1 \%$ for all the data, even for the smallest $\omega$ and largest $R e_{a}$ values. This is particularly good, as the uncertainties in the experiments were estimated to be $\lesssim 3 \%$.

\subsection{Comparison with a narrow gap approximation}

$\mathrm{Hu} \&$ Kelly (1995) present their results from a Floquet analysis of the open flow in the narrow gap limit for the degree of stabilization due to the axial oscillations of the inner cylinder in terms of a scaled relative critical Taylor number. The Taylor number is related to $R e_{i}$ by

$$
T a=\frac{4(1-e)}{1+e} R e_{i}^{2},
$$

for $e<1$. They present their results in the form $T_{r e l}=\left(T a_{c}-T a_{0}\right) /\left(T a_{0} R e_{a}^{2}\right)$ versus $\beta$, where $T a_{c}$ is the critical Taylor number for a given $R e_{a}$ and $\omega, T a_{0}$ is the critical Taylor number for $R e_{a}=0$, and $\beta=(\omega / 2)^{1 / 2}$. Hu \& Kelly find that for $R e \leqslant 30$, the curves of $T_{\text {rel }}$ versus $\beta$ are almost the same. In figure 12, we present $\mathrm{Hu} \&$ Kelly's data for $R e_{a}=30$ along with our results from figure 5 , including the $n \neq 0$ modification in the enclosed flow from figure 10, translated into $T_{r e l}$ versus $\beta$. It is clear that the narrow gap approximation is everywhere close to the open flow solution. Both the finite and narrow gap open flows grossly underestimate the level of stabilization in comparison to the experimental results (which the enclosed flow matches to within $1 \%$, see figure 11 ), and the disagreement is greatest for small $\omega$.

In the open flow, there exists a distinguished frequency above which increases in 


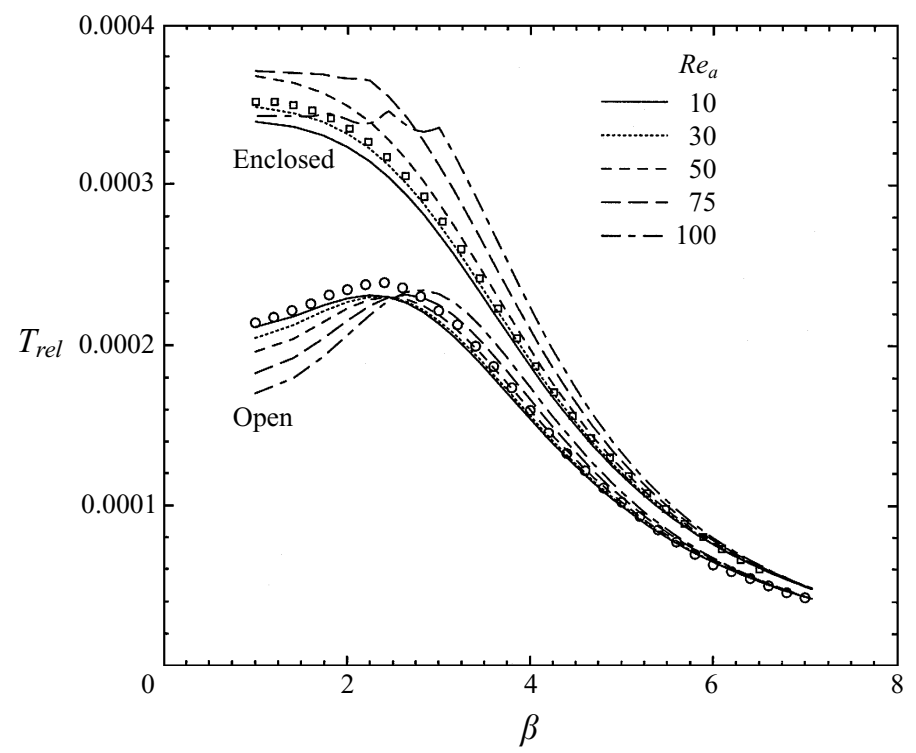

FIGURE 12. $T_{\text {rel }}$ versus $\beta$ for the open narrow gap results of $\mathrm{Hu} \&$ Kelly (1995) with $R e_{a}=30$ (circles), and the enclosed narrow gap results of $\mathrm{Hu} \&$ Kelly (private communication) with $R e_{a}=30$ (squares), together with the results from the present investigation of the open and enclosed flows for $R e_{a}$ as indicated.

$R e_{a}$ augments the degree of relative stabilization $T_{r e l}$, and below which increases in $R e_{a}$ decreases $T_{r e l}$. The frequencies that give the most stabilization in absolute terms for a given $R e_{a}$ are very close to this distinguished frequency. The degree of relative stabilization decreases markedly as $R e_{a}$ increases and $\omega \rightarrow 0$, whereas for large $\omega$ the degree of relative stabilization is independent of $R e_{a}$. In contrast, the enclosed flow does not have a corresponding distinguished frequency. The degree of relative stabilization continues to increase as both $R e_{a}$ increases and $\omega$ decreases, up to the point where the modal interactions and switches come into play. The flattening of the curves for $R e_{a}=75$ and 100 suggests a saturation of the degree of stabilization as $\omega \rightarrow 0$.

\subsection{The singular limit $\omega \rightarrow 0$}

Two interesting limits in parameter space are worth further consideration. The limit $R e_{a} \rightarrow 0$, for any $\omega$, is well behaved and simply corresponds to the usual TaylorCouette flow. For $e=0.905, R e_{i} \rightarrow 134.9$ as $R e_{a} \rightarrow 0$. This is the point from where all the curves in figure 5 emanate. The corresponding value of $k$ is 3.129. The other limit is $\omega \rightarrow 0$ for any $R e_{a}$. This limit is singular.

From a physical point of view, the singularity of the $\omega \rightarrow 0$ limit is related to the fact that the period $(2 \pi / \omega)$ of the oscillation becomes infinite. During a part of the period disturbances may be amplified and grow to amplitudes where the linearization is no longer valid. Depending on the size of initial perturbations, these large amplification rates may be viewed as transient instabilities in experiments or nonlinear numerical computations (Davis 1976; Barenghi \& Jones 1989; Murray et al. 1990). From a mathematical point of view, the $\omega \rightarrow 0$ limit results in a degenerate bifurcation where the structure of the normal form describing the bifurcation at the critical $R e_{i}$ must, to leading order, be determined by a nonlinear balance (Davis \& Rosenblat 1977). 

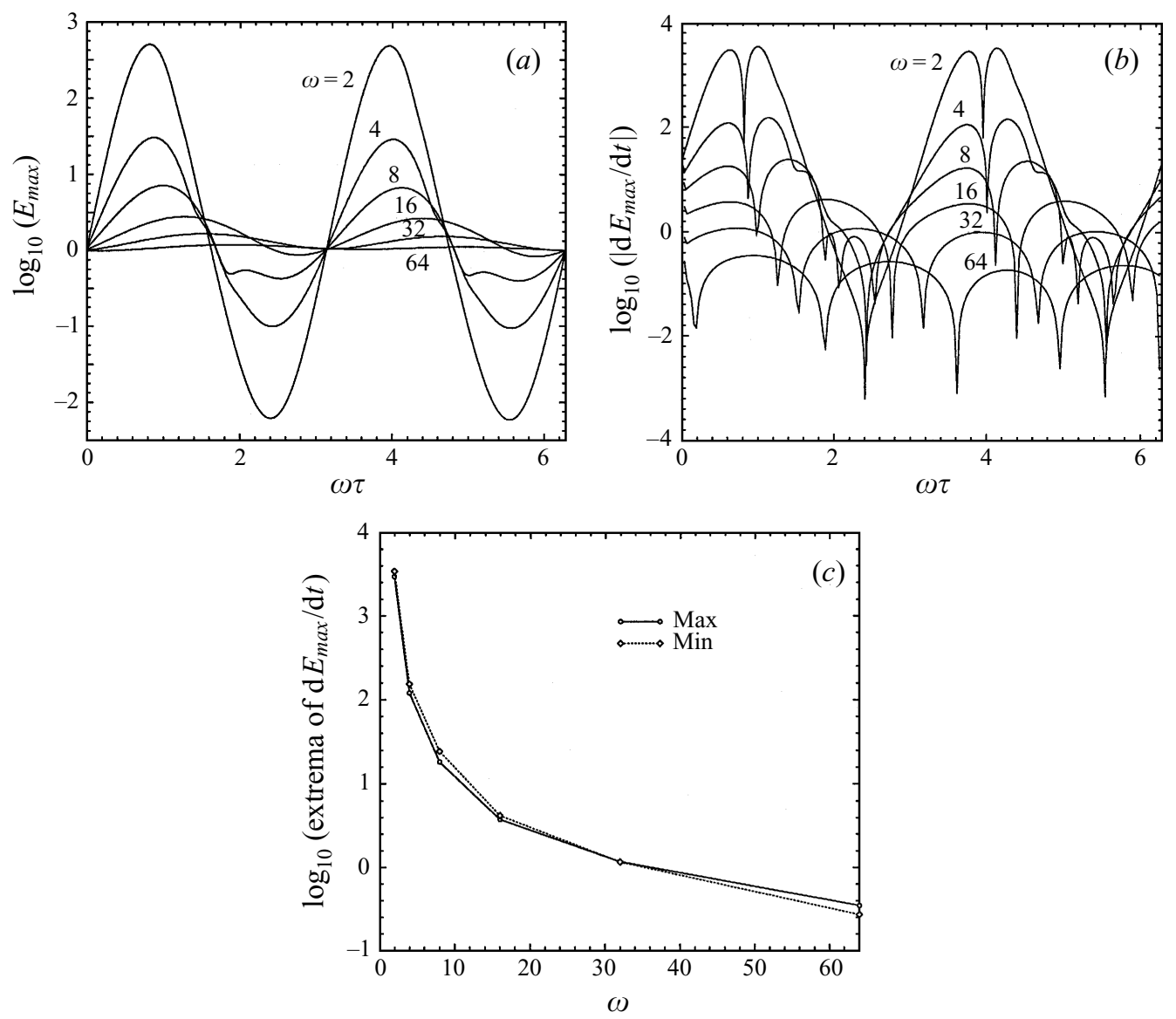

FIGURE 13. $(a) \log _{10}\left(E_{\max }(t)\right)$ versus $\omega t$ and $(b) \log _{10}\left(\left|\partial E_{\max } / \partial t\right|\right)$ versus $\omega t$ for $R e_{a}=100$ and $\omega$ as indicated in the enclosed flow, and $(c)$ the corresponding $\log _{10}$ of the absolute values of the maxima and minima of $\partial E_{\max } / \partial t$ over a period for the various $\omega$ in $(b)$.

The growth rates of initial disturbances at the bifurcation point, at any time $t$ during the period of the external forcing, can be obtained. We do this by computing the eigenvalues of the matrix $\boldsymbol{X}(t)$ from the system (3.17) and at each time step we obtain the modulus of the largest eigenvalue, $E_{\max }(t)$. The instantaneous growth rate is given by $\partial E_{\max } / \partial t$ at time $t$. Figure 13 plots $\log _{10} E_{\max }$ and $\log _{10}\left|\partial E_{\max } / \partial t\right|$ for $R e_{a}=100, e=0.905$ and various $\omega=2^{n}, n \in[1,6]$. As $\omega$ decreases, the growth rates increase dramatically. This is reflected in figure $13(c)$, plotting $\log _{10}$ of the maximum positive and negative growth rates over the period versus $\omega$. Note that the variations in the growth rates at these bifurcation points are approximately symmetric over the period, i.e. growth of disturbances is accounted for, almost symmetrically, by decay of disturbances over the whole period. The linear Floquet analysis for determining the critical $R e_{i}$ is valid, no matter how large the maximum growth rate becomes, so long as it is finite and the initial disturbances are sufficiently small that they are not amplified beyond the limit where nonlinearities come into play. However, in any physical experiment or floating point computation, there is always a minimum threshold of noise or round-off error, so that in the limit $\omega \rightarrow 0$, the instantaneous growth rates become so large that linear theory no longer applies. Given the low 
values of $\omega$ obtained in the experiment of Weisberg et al. (1997), $\omega \sim 0.5$, and the agreement with the present Floquet analysis (figure 11), this suggests that the noise level in the experiments is very low.

\section{Conclusions}

The stabilization of Taylor-Couette flow by the axial oscillation of the inner cylinder, observed experimentally by Weisberg et al. (1997), has been studied by means of Floquet theory. We have derived a closed form solution for the basic flow, independent of the axial and azimuthal directions, in an annulus of infinite length but of a finite gap, in two flow situations. These two represent an annulus with (enclosed) or without (open) endwalls. When the presence of endwalls is included in the analysis, agreement for the critical rate of rotation of the inner cylinder, $R e_{i}$, for the onset of centrifugal instability with the experiments of Weisberg et al. is achieved to well within experimental uncertainty. The open flow stabilizes the Taylor-Couette flow to a significantly lesser degree. For the gap considered here and in the experiments, curvature effects are small.

In the open flow, there is a distinguished frequency above which increases in the amplitude of the axial oscillation, $R e_{a}$, result in increases of the degree of relative stabilization, $T_{r e l}$. For oscillations at frequencies below this frequency, increases in $R e_{a}$ decreases $T_{r e l}$. In contrast, for the enclosed flow no such distinguished frequency exists, $T_{r e l}$ is increased with increasing $R e_{a}$ for all $\omega$, except in a narrow window of parameter space with $R e_{a}$ large and $\omega$ small. In this parameter regime, beyond that reported in the experiments and specifically for $R e_{a}>70$ and $\omega \sim 10$, we have found narrow windows where interactions and switching between different axial and azimuthal modes takes place. These modal interactions have only been observed for the enclosed flow and are related to a saturation of the $T_{r e l}$ versus $\omega$ relationship as $\omega \rightarrow 0$.

The analysis of the basic flow suggests that the stabilization is due to waves of azimuthal vorticity propagating out from the boundary layer on the inner cylinder in both the open and enclosed flows. Further, for the enclosed flow, waves of azimuthal vorticity also propagate inwards from the outer cylinder. These waves act to nullify the azimuthal vorticity associated with the onset of centrifugal instability of the circular Couette flow.

We would like to express our appreciation to Professor Lex Smits and Dr Arel Weisberg for the discussions and making their experimental data available, and to Professor Bob Kelly and Dr Hsiang-Cheng Hu for providing their narrow gap data for figure 12. We also like to thank Alvar Meseguer for producing figure 1. This work was partially supported by NSF grant DMS-9512483, DGICYT grant PB94-1209, and during F.M.'s visit to Penn State by DGICYES grant PR95-425.

\section{Appendix}

The linear system (3.7) is of the form $\boldsymbol{g} \dot{\boldsymbol{a}}=\boldsymbol{h}(t) \boldsymbol{a}$ where $\boldsymbol{a}$ is a complex vector and $\boldsymbol{g}, \boldsymbol{h}$ are the complex matrices

$$
\begin{aligned}
& g_{j, l}=\left\langle\tilde{\boldsymbol{u}}_{j}, \boldsymbol{u}_{l}\right\rangle, \\
& h_{j, l}=\left\langle\tilde{\boldsymbol{u}}_{j}, \Delta \boldsymbol{u}_{l}-\boldsymbol{v}_{B} \cdot \nabla \boldsymbol{u}_{l}-\boldsymbol{u}_{l} \cdot \nabla \boldsymbol{v}_{B}\right\rangle .
\end{aligned}
$$


The real version (3.16) of this system, is obtained introducing a real vector $\boldsymbol{x}$

$$
a_{j}=x_{2 j-1}+\mathrm{i} x_{2 j}
$$

and real matrices $\boldsymbol{G}, \boldsymbol{H}$ given by

$$
\begin{aligned}
& G_{2 j, 2 l}=G_{2 j-1,2 l-1}=\operatorname{Reg}_{j, l}, \\
& G_{2 j, 2 l-1}=-G_{2 j-1,2 l}=\operatorname{Im} g_{j, l}, \\
& H_{2 j, 2 l}=H_{2 j-1,2 l-1}=\operatorname{Re} h_{j, l}, \\
& H_{2 j, 2 l-1}=-H_{2 j-1,2 l}=\operatorname{Im} h_{j, l} .
\end{aligned}
$$

Introducing the basis (3.8), (3.9) in the expansion (3.6), we have

$$
\boldsymbol{u}=\sum_{j=1}^{N} a_{2 j-1} \boldsymbol{u}_{2 j-1}+a_{2 j} \boldsymbol{u}_{2 j},
$$

and after some algebra we obtain the non-zero elements of these matrices:

$$
\begin{aligned}
G_{4 i-3,4 j-3}= & G_{4 i-2,4 j-2}=\left\langle\mathrm{D}_{+} \tilde{f}_{i} \mathrm{D}_{+} f_{j}+k^{2} \tilde{f}_{i} f_{j}\right\rangle, \\
G_{4 i-2,4 j-1}= & -G_{4 i-3,4 j}=\left\langle\frac{n}{r} \mathrm{D}_{+} \tilde{f}_{i} g_{j}\right\rangle, \\
G_{4 i-1,4 j-2}= & -G_{4 i, 4 j-3}=\left\langle\frac{n}{r} \tilde{g}_{i} \mathrm{D}_{+} f_{j}\right\rangle, \\
G_{4 i-1,4 j-1}= & G_{4 i, 4 j}=\left\langle\left(k^{2}+\frac{n^{2}}{r^{2}}\right) \tilde{g}_{i} g_{j}\right\rangle, \\
H_{4 i-3,4 j-3}= & H_{4 i-2,4 j-2}=\left\langle\mathrm{D}_{+} \tilde{f}_{i}\left(\mathrm{D}_{+} \mathrm{D}-k^{2}-\frac{n^{2}}{r^{2}}\right)\right\rangle \mathrm{D}_{+} f_{j} \\
& +k^{2} \tilde{f}_{i}\left\langle\left(\mathrm{D}_{+} \mathrm{D}-k^{2}-\frac{n^{2}}{r^{2}}\right) f_{j}\right\rangle, \\
H_{4 i-3,4 j-2}= & -H_{4 i-2,4 j-3}=\left\langle\left(k w_{B}+\frac{n}{r} v_{B}\right)\left(\mathrm{D}_{+} \tilde{f}_{i} \mathrm{D}_{+} f_{j}+k^{2} \tilde{f}_{i} f_{j}\right)\right. \\
& \left.-k w_{B}^{\prime} \mathrm{D}_{+} \tilde{f}_{i} f_{j}\right\rangle, \\
H_{4 i-3,4 j-1}= & H_{4 i-2,4 j}=\left\langle\frac{n}{r}\left(k w_{B}+\frac{n}{r} v_{B}\right) \mathrm{D}_{+} \tilde{f}_{i} g_{j}+\frac{2 k^{2}}{r} v_{B} \tilde{f}_{i} g_{j}\right\rangle, \\
H_{4 i-3,4 j}= & -H_{4 i-2,4 j-1}=\left\langle-n \mathrm{D}_{+} \tilde{f}_{i}\left(\mathrm{D}_{+} \mathrm{D}-k^{2}-\frac{n^{2}}{r^{2}}\right) \frac{1}{r} g_{j}+2 n \frac{k^{2}}{r^{2}} \tilde{f}_{i} g_{j}\right\rangle, \\
H_{4 i-1,4 j-3}= & H_{4 i, 4 j-2}=\left\langle-\frac{n}{r}\left(k w_{B}+\frac{n}{r} v_{B}\right) \tilde{g}_{i} \mathrm{D}_{+} f_{j}\right. \\
& \left.+\left(\frac{n k}{r} w_{B}^{\prime}-k^{2} \mathrm{D}_{+} v_{B}\right) \tilde{g}_{i} \mathrm{D}_{+} f_{j}\right\rangle, \\
H_{4 i-1,4 j}= & -H_{4 i, 4 j-1}=\left\langle\left(k^{2}+\frac{n^{2}}{r^{2}}\right)\left(k w_{B}+\frac{n}{r} v_{B}\right) \tilde{g}_{i} g_{j}\right\rangle, \\
H_{4 i, 4 j-3}= & -H_{4 i-1,4 j-2}=\left\langle-\frac{n}{r} \tilde{g}_{i}\left(\mathrm{D}_{+} \mathrm{D}-k^{2}-\frac{n^{2}}{r^{2}}\right) \mathrm{D}_{+} f_{j}+2 n \frac{k^{2}}{r^{2}} \tilde{g}_{i} f_{j}\right\rangle, \\
H_{4 i-1,4 j-1}= & H_{4 i, 4 j}=\left\langle\tilde{g}_{i}\left(k^{2} \mathrm{DD}_{+} g_{j}+\frac{n^{2}}{r^{2}} \mathrm{D}_{+} \mathrm{D}_{\frac{1}{r} g_{j}}\right)-\left(k^{2}+\frac{n^{2}}{r^{2}}\right)^{2} \tilde{g}_{i} g_{j}\right\rangle, \\
= &
\end{aligned}
$$


where a $k^{2}$ factor has been introduced, and \langle\rangle means integration with respect to $r$ :

$$
\langle f\rangle=\int_{r_{i}}^{r_{o}} f(r) r \mathrm{~d} r .
$$

\section{REFERENCES}

Abramowitz, M. \& Stegun, I. 1972 Handbook of Mathematical Functions. Dover.

Ali, M. E. \& Weidman, P. D. 1993 On the linear stability of cellular spiral Couette flow. Phys. Fluids A 5, 1188-1200.

Barenghi, C. F. \& Jones, C. A. 1989 Modulated Taylor-Couette flow. J. Fluid Mech. 208, 127-160.

Canuto, C., Hussaini, M. Y., Quarteroni, A. \& Zang, T. A. 1988 Spectral Methods in Fluid Dynamics. Springer.

CARMi, S. \& Tustaniwskyj, J. I. 1981 Stability of modulated finite-gap cylindrical Couette flow: linear theory. J. Fluid Mech. 108, 19-42.

Chossat, P. \& Iooss, G. 1994 The Couette-Taylor Problem. Springer.

Davis, S. H. 1976 The stability of time-periodic flows. Ann. Rev. Fluid Mech. 8, 57-74.

Davis, S. H. \& Rosenblat S. 1977 On bifurcating periodic solutions at low frequency. Stud. Appl. Maths 57, 59-76.

DonNelly, R. J. 1964 Experiments on the stability of viscous flow between rotating cylinders. III. Enhancement of stability by modulation. Proc. R. Soc. Lond. A 281, 130-139.

Donnelly, R. J., ReIF, F. \& SuHL, H. 1962 Enhancement of hydrodynamic stability by modulation. Phys. Rev. Lett. 9, 363-365.

Edwards, W. S., Tagg, R. P., Dornblaser, B. C. \& Swinney, H. L. 1991 Periodic traveling waves with nonperiodic pressure. Eur. J. Mech. B Fluids 10, 205-210.

Guckenheimer, J. \& Holmes, P. 1986 Nonlinear Oscillations, Dynamical Systems, and Bifurcations of Vector Fields. Springer.

Hairer, E. \& Wanner, G. 1991 Solving Ordinary Differential Equations. II. Stiff and DifferentialAlgebraic Problems. Springer.

Hu, H. C. \& Kelly, R. E. 1995 Effect of a time-periodic axial shear flow upon the onset of Taylor vortices. Phys. Rev. E 51, 3242-3251.

IsaAcson, E. \& Keller, H. B. 1966 Analysis of Numerical Methods. John Wiley \& Sons.

JoSEPH, D. D. 1976 Stability of Fluid Motions I. Springer.

KERCZEK, C. vON. \& DAVIS, S. H. 1974 Linear stability theory of oscillatory Stokes layers. J. Fluid Mech. 62, 753-773.

Moser, R. D., Moin, P. \& Leonard, A. 1983 A spectral numerical method for the Navier-Stokes equations with applications to Taylor-Couette Flow. J. Comput. Phys. 52, 524-544.

Murray, B. T., McFadden, G. B. \& Coriell, S. R. 1990 Stabilization of Taylor-Couette flow due to time-periodic outer cylinder oscillation. Phys. Fluids A 2, 2147-2156.

Sanchez, J., Crespo, D. \& Marques, F. 1993 Spiral vortices between concentric cylinders. Appl. Sci. Res. 51, 55-59.

Swinney, H. L. \& Gollub, J. P. 1985 Hydrodynamic Instabilities and the Transition to Turbulence. Springer.

Weisberg, A. Y., Kevrekidis, I. G. \& Smits, A. J. 1997 Delaying transition in Taylor-Couette flow with axial motion of the inner cylinder. J. Fluid Mech. 348, 141-151. 